\title{
Pressure-volume properties of endlinked hard-chain polymer networks
}

\author{
N. R. Kenkare, C. K. Hall, a) and S. A. Khan \\ Department of Chemical Engineering, Box 7905, North Carolina State University, Raleigh, \\ North Carolina 27695-7905
}

(Received 5 November 1998; accepted 22 January 1999)

\begin{abstract}
Equilibrium molecular dynamics simulations are used to obtain the pressure and configurational chain properties of near-perfect, off-lattice, trifunctional hard-chain networks of chain lengths 20 , 35,50 , and 100, and of tetrafunctional hard-chain networks of chain lengths 20, 35, and 50 over a range of packing fractions. Our simulation results show that the variation of network pressure with density is similar to that of uncrosslinked chain systems of the same chain length, except at low densities where the network pressure shows a negative region, as first observed by Escobedo and de Pablo. We present a theoretical treatment leading to an analytical expression for the network pressure as the sum of liquid-like and elastic contributions. The liquid-like contribution is obtained by extending the generalized Flory-dimer theory to networks, and the elastic contribution is obtained by treating the network as a set of interpenetrated dendrimers and using an ideal chain-spring analogy to calculate the free energy. The theoretical predictions for network pressure are in good agreement with simulation data. (C) 1999 American Institute of Physics.
\end{abstract}

[S0021-9606(99)51615-2]

\section{INTRODUCTION}

The immense technological importance of polymer networks and gels in the automobile, plastics, and medical equipment industries is due primarily to their striking elastic properties. As a consequence, a great deal of theoretical and experimental research has been conducted over the past fifty years in an attempt to understand the nature of the stressstrain relations of polymer networks.

In this article, we investigate the molecular mechanisms underlying polymer network behavior by performing discontinuous molecular dynamics simulations. A comprehensive study of the pressure-volume properties of trifunctional and tetrafunctional networks is conducted and the resulting data are used to evaluate the predictions of several existing network theories. We also propose an analytical mean-field network equation of state based on a simple network model; this equation of state yields good agreement with the data from our simulations.

The earliest molecularly based theories for crosslinked polymers are the affine model of Flory and Rehner, ${ }^{1}$ and Wall, ${ }^{2}$ and the phantom model of James and Guth. ${ }^{3,4}$ Both models are based on mean-field approaches in which the analogy between the elastic properties of an ideal, Gaussian chain and a classical elastic spring is used to calculate the network free energy. By considering the network to be a collection of such chains, assuming that the chain vectors deform proportionally with macroscopic deformation (the affine assumption), and neglecting excluded volume interactions, the affine and phantom models arrive at a simple stress-strain relationship for network deformation. These

\footnotetext{
a) Author to whom correspondence should be addressed; electronic mail: hall@turbo.che.ncsu.edu
}

models are successful in predicting the behavior of real networks at small deformations, but are unsuccessful at large deformations.

A number of other theories of network behavior have been developed in an attempt to overcome some of the shortcomings of the affine and phantom models. These include the constrained junction models of Flory and Erman, ${ }^{5,6}$ and Ronca and Allegra ${ }^{6,8}$ as well as several localization, slip-link and tube models. ${ }^{7-22}$ These theories partially account for the excluded volume interactions in networks (which are neglected by the affine and phantom models), and have been qualitatively successful in predicting the stress-strain relation of real networks in the small and intermediate deformation regimes.

Much of the theoretical work on crosslinked systems applies to 'perfect', networks, i.e., networks with constant chain length between the junctions and few structural irregularities (dangling chains, loops, or junctions of varying functionality). The validation of network theories is best done using "model" near-perfect networks with wellcharacterized molecular structure. Experimental efforts in this direction are, however, hampered by the difficulty associated with obtaining precise information about the network structure. In the last decade, computer simulations have proved to be an excellent method for investigating network behavior since they allow the investigator to construct and study near-perfect model networks with well-characterized structure.

Most simulation studies of polymer networks to date have focussed on the kinetics of crosslinking or on network dynamics. ${ }^{23-31}$ Simulation studies of the pressure-volumetemperature $(P-V-T)$ properties of networks are relatively rare, even though such investigations would yield valuable insights into the elastic response of a network undergoing 
isotropic deformation, and would enhance our understanding of the molecular mechanisms underlying gel swelling.

To our knowledge, the only investigations of the $P-V-T$ behavior of networks are those of Escobedo and de Pablo, ${ }^{32-34}$ and Sommer. ${ }^{35}$ Escobedo and de Pablo conducted NPT ensemble (constant number of particles, $N$, pressure, $P$, and temperature, $T$ ) Monte Carlo simulations of short chain (chain lengths from 1 to 32), tetrafunctional, athermal, and square-well networks having a diamond-like junction connectivity. The networks were created in such a way that the systems contained no trapped entanglements. Owing to the limitations of conventional Monte Carlo methods for simulating branched and crosslinked systems, Escobedo and de Pablo invented a set of complex system-specific Monte Carlo moves, the extended continuum configurational bias (ECCB), "slab," and "cluster" moves for relaxing the network and for making the volume changes necessary in a NPT simulation. They found that the $P-V$ behavior of network systems did not differ substantially from that of linear or star polymers except at very low packing fractions where a region of negative pressure was seen. They also presented a semitheoretical method to calculate the network free energy from the sum of the free energy of a system of reference polymer molecules (e.g., octahedral molecules) and the free energy associated with network elasticity, thus dividing the network energy into liquid-like and elastic components as suggested originally by James and Guth. ${ }^{3}$ The elastic free energy of the network was obtained from a "computer experiment" in which the entropy of an isolated chain was calculated by enumerating all of the self-avoiding walks possible between the chain ends when they were maintained a fixed distance apart in space. This single chain entropy was then used in conjunction with the affine assumption to obtain the elastic contribution to the network free energy. The theory showed good agreement with their simulation data in the very low packing fraction regime, but overpredicted the data at intermediate to high densities. Escobedo and de Pablo's approach is an improvement over many existing network theories in that the elastic term clearly accounts for the excluded volume of the network chains. However, the fact that a set of computer simulations must be conducted at each chain length in order to calculate the elastic contribution to the network free energy means that network properties cannot be obtained analytically. This precludes the use of this approach for predicting the properties of an arbitrary network.

Sommer $^{35}$ conducted bond-fluctuation Monte Carlo simulations of a large randomly crosslinked athermal network consisting of approximately 50000 segments arranged on a cubic lattice. To simulate an isotropic deformation process, the network was simply allowed to diffuse into a larger lattice, and the chain deformation and the network pressure were calculated as a function of the resulting macroscopic deformation of the network. Sommer observed that the network chain deformation was dependent on molecular weight, i.e., the shorter chains in the randomly crosslinked network were generally less deformed than the longer chains at a given macroscopic deformation. The pressure of the crosslinked system was observed to be different from that of an uncrosslinked system particularly at large deformations; the difference in pressures was related to the disparity in the fractal-like properties of the two systems.

Our study of network behavior is motivated partly by the paucity of simulation data on the $P-V-T$ and structural properties of crosslinked systems, and partly by the need for an analytical network equation of state. The purpose of this article is to investigate the pressure-volume properties of model athermal trifunctional and tetrafunctional networks using equilibrium molecular dynamics simulation techniques, and to propose an analytical equation of state for networks that is valid over a wide range of densities.

We use discontinuous molecular dynamics simulations to construct near-perfect, off-lattice, monodisperse, trifunctional hard-chain networks (i.e., networks in which the chains consist of strings of hard spheres) of chain lengths 20 , 35,50 , and 100 , and tetrafunctional hard-chain networks of chain lengths 20,35 , and 50 . The networks are created from melts of linear tangent hard-sphere chains (chains in which successive hard-sphere segments are tangential to each other) by endlinking, i.e., by allowing bond formation between chain ends that collide with each other in the course of the simulation, up to a maximum of two bonds per chain end in a trifunctional network or three bonds per chain end in a tetrafunctional network. Our crosslinking process is similar to the experimental procedure used to develop "room temperature vulcanizates," as well as the method used by a number of researchers ${ }^{36-39}$ to synthesize model networks with a known chain molecular weight distribution between crosslinks. Even for these model experimental systems, however, the difficulty of obtaining a sufficiently monodisperse precursor system remains. In addition, it is difficult to obtain a precise knowledge of the extent of crosslinking of the system, and the proportion of irregularities, which is necessary in order to investigate the thermophysical properties of networks. In comparison, computer simulations offer a greater control over the crosslinking process, allowing us to create monodisperse networks (with a constant chain length between junctions), as well as giving us a precise knowledge of the network structure.

In our systems, the $P-V$ and structural properties of the endlinked networks are investigated over a range of packing fractions $(0.45-0.005)$ by expanding the network from its initial packing fraction of 0.45 to successively lower densities. The network expansion is done by using an algorithm that utilizes the variable bond-length attribute of the Bellemans chain to conduct isotropic volume changes. The advantage of using this algorithm is that it allows us to simulate network expansion/contraction without introducing complex, system-specific moves, and without imposing an affine transformation on the chain ends.

Highlights of our simulation results are the following. We observe that the network pressure follows the same trend as that for uncrosslinked chain systems over packing fractions from $\eta=0.45$ to about $\eta=0.1$, i.e., the pressure decreases continuously with decreasing packing fraction. At low packing fractions $(\eta<0.1)$ the network pressure becomes negative, in contrast to the behavior of uncrosslinked chain systems. As the packing fraction is lowered even fur- 
ther $(\eta<0.01)$, there is a sharp downturn in the network pressure, that is, the pressure drops suddenly towards the highly negative region. These observations are in agreement with the results of Escobedo and de Pablo. ${ }^{33,34}$ We find that the sharp downturn in network pressure observed at very low packing fractions occurs when the average end-to-end distance of the network chains is between $50 \%$ and $70 \%$ of its maximum extended length. We also observe that the $P-V$ properties and chain dimensions of tetrafunctional networks do not differ appreciably from those of trifunctional networks.

We present a model based in statistical thermodynamics that predicts the pressure-volume behavior of athermal polymer networks. The network is modeled as a set of interpenetrated dendrimers, whose branches are the network chains. Following James and Guth, ${ }^{3}$ the network pressure at a given packing fraction is taken to be the sum of a liquid-like term which arises from the repulsive part of the intermolecular interaction, and an elastic term which arises from the retractive force exerted by the chains opposing the network deformation. The liquid-like term is obtained from the generalized Flory-Dimer equation of state ${ }^{40-42}$ by considering each dendrimer to be made up of a number of connected star-like molecules. The elastic term is obtained by calculating the free energy of a dendrimer in terms of the free energies of its component chains using the simple ideal-chain/spring analogy. The dendrimer chains are treated in two ways: (1) as having a Gaussian chain vector distribution (the Gaussian dendrimer model), and (2) as having a non-Gaussian chain vector distribution of the form suggested by Kuhn and Grun $^{43,44}$ (the non-Gaussian dendrimer model). We find that while the Gaussian dendrimer model predicts the network pressures fairly well over a wide range of packing fractions, it is unable to predict the sudden drop in pressure that occurs at very low densities. In comparison, the non-Gaussian dendrimer model predicts the network pressures over the entire range of packing fractions and is remarkably successful in the extremely low density region.

The organization of our article is as follows: Section II describes the molecular model and the general simulation technique, as well as the simulation methods used for network construction and for network volume changes. In Sec. III, our theoretical model is outlined, and in Sec. IV, our simulation and theoretical results are discussed. A brief summary and some further discussion are given in Sec. V.

\section{MOLECULAR MODEL AND SIMULATION TECHNIQUE}

In this article, the discontinuous molecular dynamics technique that we have used previously to treat polymer melts ${ }^{45,46}$ has been adapted to the case in which linear melt chains are endlinked to form a polymer network. We begin this section by reviewing the discontinuous molecular dynamics technique as it is applied to a polymer melt. We then outline our method for constructing and relaxing polymer networks, and finally we describe our technique for conducting network volume change moves.

The basic unit of the polymer network is a polymer chain which we represent as a freely jointed tangent hard- sphere chain. The potential energy of interaction between any two beads on the same or neighboring chains in the network is given by

$$
U(r)=\left\{\begin{array}{cc}
0, & r>\sigma \\
\infty, & r \leqslant \sigma,
\end{array}\right.
$$

where $r$ is the bead-bead distance and $\sigma$ is the bead diameter.

The treatment of a system of tangent hard sphere chains using standard molecular dynamics techniques is difficult due to the tangency requirement and flexible nature of such chains. To circumvent this problem, Rapaport ${ }^{47,48}$ and Bellemans, Orban, and Belle ${ }^{49}$ suggested an approach in which the bond length, $l$, between successive segments of the chain is not kept constant, but is instead allowed to vary freely over a range, $\sigma(1-\delta)<l<\sigma(1+\delta)$ where $\delta \ll 1$. The chain then effectively becomes a collection of segments connected by sliding links (the Bellemans chain). As $\delta \rightarrow 0$, the Bellemans chain approximates the tangent hard-sphere chain model. The relaxation of the constant bond length requirement allows the chain segment trajectories to be partially decoupled, resulting in linear trajectories between collisions. The system dynamics can then be treated using the discontinuous molecular dynamics techniques developed by Alder and Wainwright ${ }^{50-52}$ for hard-sphere systems.

Molecular dynamics simulations of systems with discontinuous potentials, such as the hard-sphere potential, take advantage of the fact that the equations of motion for these systems can be solved analytically at successive "collisions." A collision (or event) occurs when the distance between any two segments becomes equal to a point of discontinuity in the potential. Thus the dynamics of a system of molecules governed by the hard-sphere potential evolves on a collision-by-collision basis. For a Bellemans chain, collisions can be of two types: core collisions and bond stretches. Core collisions can occur between any pair of segments, $i$ and $j$, in the system when the distance between two bonded segments becomes equal to $\sigma(1-\delta)$, or when the distance between two nonbonded segments becomes equal to $\sigma$. Bond stretches occur between pairs of bonded segments when the distance between the segments becomes equal to the maximum stretch of the Bellemans bond, $\sigma(1+\delta)$. The simulation proceeds by locating the next event, advancing all the segments until the event occurs, and calculating collision dynamics for the colliding pair of segments. This process is performed repeatedly. A number of time-saving techniques are used to improve the algorithm efficiency based on the work of Smith, Hall, and Freeman, ${ }^{46}$ including neighbor lists, linked lists, binary trees, and delayed position updates.

In order to construct trifunctional or tetrafunctional networks, we endlinked melts of linear Bellemans chains. The endlinking was done at an initial system packing fraction of $\eta=0.3$ after which the packing fraction was increased to $\eta$ $=0.45$, where $\eta$ is defined by

$$
\eta=\frac{\pi}{6} \frac{N_{c} n \sigma^{3}}{V}
$$

with $N_{c}$ equal to the number of network chains, $n$ equal to the chain length, and $V$ equal to the volume of the simulation cell. We adopted this procedure (see our previous 
TABLE I. The chain length, $n$, number of chains, $N_{c}$ and the total number of segments, $N_{t}$ for each simulation system.

\begin{tabular}{lrrrrrrr}
\hline \hline & \multicolumn{3}{c}{ Trifunctional } & \multicolumn{4}{c}{ Tetrafunctional } \\
\hline$n$ & 20 & 35 & 50 & 100 & 20 & 35 & 50 \\
$N_{c}$ & 90 & 90 & 90 & 90 & 120 & 120 & 120 \\
$N_{t}$ & 1800 & 3150 & 4500 & 9000 & 2400 & 4200 & 6000 \\
\hline \hline
\end{tabular}

publication $^{30}$ for details) because it is relatively easy to achieve near-complete extents of reaction at lower densities, particularly for the longer chains. This crosslinking procedure is found to impact network elastic properties, causing the elastic behavior of networks that are crosslinked at low densities and then compressed to the (high) density of interest to be somewhat different from those of networks that are crosslinked at high densities. ${ }^{30}$ This is attributed to topological differences between these networks; for instance, networks that are crosslinked at low initial densities are less interpenetrated and contain fewer trapped entanglements than networks crosslinked at high densities. Also the chains in the networks crosslinked at low densities are somewhat collapsed relative to the chains in the networks crosslinked at high densities, even after the system density is increased. The decreased chain dimensions and changed entanglement topology associated with networks crosslinked at low densities are expected to affect the elastic properties of these networks so that they have "superelastic" properties (see Obhukov, Rubinstein, and Colby ${ }^{53}$ ). That is, networks crosslinked at low initial density (which are akin to solutioncrosslinked experimental systems from which the solvent is extracted) can be elongated to much larger deformations before they rupture than networks crosslinked at a high initial density (which are akin to melt-crosslinked networks). Although we have chosen a fairly high initial density for crosslinking $(\eta=0.3)$, in order to ensure that the network topology is not greatly affected by our crosslinking procedure, we expect that the network's elastic properties will be influenced to some extent.

In this article, four trifunctional networks of chain lengths 20, 35, 50, and 100 and three tetrafunctional networks of chain lengths 20,35, and 50 are studied. As shown in Table I, each trifunctional network contains 90 chains, and each tetrafunctional network contains 120 chains, the numbers being chosen so that the number of junctions, $N_{f}$, in all of the networks is approximately the same $\left(N_{f}=2 N_{c} / f\right.$ $\sim 60$, where $f$ is the network functionality). The chain segment diameter, $\sigma=1$, the Bellemans bond variation factor, $\delta=0.05$, and the box length varies with the packing fraction. The structural characteristics of the networks, displayed in Table II, are described in terms of the elastically active fraction, $\%_{\text {elastic }}$, the number of trifunctional junctions, $N_{3}$, the number of tetrafunctional junctions, $N_{4}$, the number of bifunctional (unsaturated) junctions, $N_{\text {unsat }}$, the number of dangling ends and loops, $N_{\text {end }}$ and $N_{\text {loop }}$, and the number of free chains, $N_{\text {free }}$. The elastically active fraction is an important measure of the "perfectness" of our networks, and is defined to be the fraction of chains that are connected at both ends to junctions with at least three independent paths to the
TABLE II. The network elastic fraction, $\%_{\mathrm{el}}$, the number of trifunctional and tetrafunctional junctions, $N_{3}$ and $N_{4}$, the number of unsaturated junctions, $N_{\text {unsat }}$, the number of dangling ends, $N_{\text {end }}$, the number of loops, $N_{\text {loop }}$, and the number of free chains, $N_{\text {free }}$ in the trifunctional and tetrafunctional network systems.

\begin{tabular}{|c|c|c|c|c|c|c|c|}
\hline \multirow[b]{2}{*}{$\mathrm{N}$} & \multicolumn{4}{|c|}{ Trifunctional } & \multicolumn{3}{|c|}{ Tetrafunctional } \\
\hline & 20 & 35 & 50 & 100 & 20 & 35 & 50 \\
\hline$\%_{\mathrm{el}}$ & 0.99 & 0.96 & 0.90 & 0.84 & 0.96 & 0.94 & 0.93 \\
\hline$N_{3}$ & 60 & 59 & 58 & 57 & 3 & 4 & 7 \\
\hline$N_{4}$ & $\ldots$ & $\ldots$ & $\ldots$ & $\ldots$ & 57 & 56 & 52 \\
\hline$N_{\text {unsat }}$ & 0 & 1 & 2 & 3 & 0 & 1 & 3 \\
\hline$N_{\text {end }}$ & 0 & 2 & 2 & 3 & 3 & 2 & 5 \\
\hline$N_{\text {loop }}$ & 1 & 1 & 3 & 5 & 6 & 4 & 1 \\
\hline$N_{\text {free }}$ & 0 & 0 & 2 & 0 & 0 & 0 & 0 \\
\hline
\end{tabular}

network. ${ }^{54,55}$ It is calculated in Table II by subtracting the number of unsaturated junctions, dangling chains, and loops from the total number of network chains, and dividing by the total number of network chains. Since the elastically active fraction is close to 1 for all the networks considered, and the number of dangling chains, loops and unsaturated junctions is low, we consider these networks to be near-perfect systems.

Once the networks attained their final packing fraction of 0.45 , they were relaxed for 500 million collisions. The compressibility factors were then evaluated from the Clausius virial theorem in the following form:

$$
Z^{\text {net }} \equiv \frac{P V}{N^{\text {net }} k_{B} T}=N_{c} n-\frac{m_{s} \Sigma_{\mathrm{coll}} \mathbf{r}_{i j} \cdot \Delta \mathbf{v}_{i j}}{3 k_{B} T t_{e}},
$$

where $P$ is the network pressure, $N^{\text {net }}$ is the number of " network molecules" ( $N^{\text {net }}=1$ since the network is considered to be one large molecule), $t_{e}$ is the elapsed simulation time over which the sum is calculated, $k_{B}$ is the Boltzmann constant, $T$ is the temperature, $m_{s}$ is the mass of a segment, $\mathbf{r}_{i j}$ is the vector between segment centers at a collision, and $\Delta \mathbf{v}_{i j}$ is the velocity change for the colliding pair. In this article, the simulation results are reported mostly in terms of the reduced pressure, $P^{*} \equiv P \sigma^{3} / k_{B} T=Z^{\text {net }} N^{\text {net }} 6 \eta /\left(\pi N_{c} n\right)$. We also calculated the mean-squared end-to-end distance of the network chains, $\left\langle R^{2}\right\rangle$, which is defined by

$$
\left\langle R^{2}\right\rangle=\left\langle\left(\mathbf{r}_{1}-\mathbf{r}_{n}\right)^{2}\right\rangle,
$$

where $\mathbf{r}_{1}$ and $\mathbf{r}_{n}$ are the coordinates of the first and last chain segments, respectively, and the ensemble average is taken over all the chains in the network.

In order to obtain the properties of networks at different packing fractions, we devised a novel algorithm to isotropically expand or contract the network from its initial, equilibrated state at $\eta=0.45$ to a final deformed state at any other packing fraction. This algorithm takes advantage of the variable bond length of the Bellemans chain and allows us to conduct network volume changes without having to break and regrow the chains, and without causing segment overlaps. The procedure for network expansion is as follows: When the system undergoes a core collision, we conduct a search among all the bonded pairs of segments in the system for the pair of segments, $i$ and $j$, that have the largest inter- 
segment distance, $R_{\max }$. Since the maximum allowable distance between a pair of bonded segments is $\sigma(1+\delta)$, the bond length $l_{i j}$ between that pair, $i$ and $j$, could be increased until $l_{i j}=\sigma(1+\delta)$. Accordingly we scale the positions of all the segments in the box, as well as the box length by the factor, $\kappa=\sigma(1+\delta) / R_{\max } \geqslant 1$. This does not cause bond breakage or overlaps, since $i$ and $j$ are the bonded pair that are the furthest apart in the system. The procedure for network contraction is similar: When the system undergoes a bond stretch, we conduct a search among all bonded and unbonded pairs of segments in the system for the pair of segments, $i$ and $j$, that have the smallest intersegment distance, $R_{\min }$. Since the minimum allowable distance between a pair of segments is either $\sigma(1-\delta)$ or $\sigma$, depending on whether the segments are bonded or nonbonded, the distance $l_{i j}$ between $i$ and $j$ could be decreased until $l_{i j}=\sigma(1-\delta)$ (bonded pair) or $l_{i j}=\sigma$ (unbonded pair). Accordingly we scale the positions of all the segments in the box, as well as the box length by the factor, $\kappa=\sigma(1-\delta) / R_{\min }$, if $i$ and $j$ are bonded, or by $\kappa=\sigma / R_{\min }$ if $i$ and $j$ are nonbonded, without causing segment overlaps. After the segment positions and box length are scaled, the system neighbor lists and time lists are regenerated. This procedure is repeated every $N_{\text {coll }}$ collisions (for e.g., $N_{\text {coll }}=1000$ ), until the required packing fraction (or volume) is achieved.

The use of the algorithm described above to expand/ contract the network to the required packing fraction represents a considerable computational saving over the alternatives of either constructing a network at each required density or using complex chain breakage and regrowth moves to maintain the network topology during volume changes. This method also offers us the advantage that the network structural characteristics are the same at each density, allowing us to ignore variations in the network structure in our analysis of network static properties. The disadvantage of this algorithm is that it is inefficient when applied to large $(>10000$ segments) systems, the reason being that all of the segment positions and collision times have to be recalculated after a volume change move is carried out, causing a complete rescheduling of events.

We have used this algorithm to expand the network from an initial packing fraction of $\eta=0.45$ to successively lower packing fractions of $\eta=0.35,0.25, \ldots, 0.003$. Since the network at $\eta=0.45$ is the starting point for all expansions of the system, the network is considered to be undeformed at this packing fraction. At any lower packing fraction $(\eta<0.45)$, the network is considered to be deformed. An additional criterion on which to base the description of the $\eta=0.45$ network as being undeformed is that the mean-squared end-toend distance of the network chains at $\eta=0.45$ is equal to the mean-squared end-to-end distance of uncrosslinked system chains at the same packing fraction (this will be shown in Sec. IV). Hence the configurational entropy of the network chains at a packing fraction of 0.45 is approximately the same as that of the equivalent uncrosslinked system chains at the same packing fraction. At any lower packing fraction $(\eta<0.45)$, the mean-squared end-to-end distance of the network chains is greater than the mean-squared end-to-end distance of uncrosslinked system chains at this same $\eta$, and the configurational entropy of the network chains is lower than that of the uncrosslinked system chains, again at the same $\eta$. For all of these reasons the network is considered to be undeformed at the initial packing fraction of $\eta_{0}=0.45$. The deformation ratio of a network at any packing fraction $\eta$ $\leqslant \eta_{0}$ is defined as

$$
\lambda=\lambda_{x}=\lambda_{y}=\lambda_{z}=\left[\frac{\eta_{0}}{\eta}\right]^{1 / 3}=\left[\frac{0.45}{\eta}\right]^{1 / 3}=\left[\frac{V}{V_{0}}\right]^{1 / 3}=\frac{L}{L_{0}},
$$

where $\eta_{0}, V_{0}, L_{0}$, and $\eta, V, L$, are the packing fractions, simulation cell volumes, and lengths for the undeformed and deformed networks, respectively. Once the network has attained its final packing fraction, $\eta$, it is relaxed for 500 million collisions, and the compressibility factor and chain dimensions are calculated.

We also conducted simulations of uncrosslinked chain systems containing 20 chains each of chain lengths 20,35 , 50 , and 100, over a packing fraction range of $\eta$ $=0.45-0.01$. Our purpose in conducting these simulations was to obtain accurate data for the pressure-volume behavior and the chain dimensions of uncrosslinked systems which could be compared to our network data. The 20-mer uncrosslinked chain systems were relaxed for 100 million collisions each, and all the other systems were relaxed for 500 million collisions each. The compressibility factors were evaluated using the Clausius virial theorem in the following form:

$$
Z^{\text {chain }} \equiv \frac{P V}{N_{c} k_{B} T}=n-\frac{m_{s} \Sigma_{\text {coll }} \mathbf{r}_{i j} \cdot \Delta \mathbf{v}_{i j}}{3 N_{c} k_{B} T t_{e}} .
$$

The chain dimensions were evaluated using Eq. (4).

\section{THEORY}

In this section, we propose a new equation of state for polymer networks which is based on modeling the network as a set of interpenetrated dendritic structures. The network free energy is taken to be the sum of a liquid-like contribution and an elastic contribution. The liquid-like contribution is obtained from the generalized Flory-dimer (GFD) theory, and the elastic contribution is obtained by extending the formalism of the affine model to the dendrimer-network model. Although the assumption that the network free energy can be divided into liquid-like and elastic contributions has been the subject of debate (Gee, Herbert, and Roberts, ${ }^{56}$ Eichinger and Neuburger ${ }^{57}$ ), we have used it in this work because the simplicity of the assumption allows us to obtain an analytical expression for the network free energy.

To help us explain the physical basis for our model and to point out its similarities and differences with existing theories of network elasticity, we begin Sec. III with a brief description of the affine $e^{1,2}$ and phantom ${ }^{3,4}$ theories. Next, we outline the network theories of Duiser and Staverman, ${ }^{58}$ and of Graessley ${ }^{59}$ who modeled the polymer network as an ensemble of micronetworks, and obtained results that deviate somewhat from the affine and phantom predictions. Last, we describe our theory in detail.

In the affine and phantom theories, the polymer network is modeled as a system of ideal chains whose elastic proper- 
ties are similar to those of classical elastic springs. Thus the forces exerted by the network are the same as would be produced if each chain were replaced by an elastic spring. This means that each pair of chain ends in the affine or phantom network is under the action of a force tending to pull the chain ends together, and that, in the absence of other forces, the network would collapse to a zero volume. ${ }^{44}$ In a real rubber-like network, this tendency to collapse is balanced by the mutual repulsive forces between molecules. Thus, in a real rubber, two different forces can be distinguished (James and $\mathrm{Guth}^{3}$ ), the first being the elastic force that is related to the configurational entropy of the chains and the second being the internal "hydrostatic" or liquid-like force that is associated with the interatomic forces between the network segments. The latter force is the same as that which exists in an ordinary liquid.

We now briefly review the steps taken in the affine theory to calculate the free energy of a deformed ideal chain network when the network undergoes an isotropic expansion/ contraction from an initial volume, $V_{0}$, to a final volume, $V=\lambda^{3} V_{0}$. The theory rests on a number of premises. Each network chain is assumed to be ideal, which means that the probability distribution associated with its end-to-end vectors is Gaussian at all deformations. The probability distribution, $p(\mathbf{R})$, of the chain end-to-end vectors, $\mathbf{R}$, is expressed as the product of the probability distributions of the components of the chain end-to-end vectors along each of the coordinate axes,

$$
\begin{aligned}
p_{\text {chain }}(\mathbf{R}) & =p_{\text {chain }}(X) p_{\text {chain }}(Y) p_{\text {chain }}(Z) \\
& =C \exp \left(-\frac{3 R^{2}}{2\left\langle R_{0}^{2}\right\rangle}\right)
\end{aligned}
$$

where $C$ is a constant and $\left\langle R_{0}^{2}\right\rangle$ is the mean squared end-toend distance of the undeformed chain, which is taken to be equal to the mean squared end-to-end distance of a chain in the equivalent melt, i.e., $\left\langle R_{0}^{2}\right\rangle \equiv n \sigma^{2}$. The chain entropy, $S(R)$ is given by

$$
S_{\text {chain }}(R)=k_{B} \ln p_{\text {chain }}(\mathbf{R})=k_{B} \ln C-\frac{3 k_{B} R^{2}}{2\left\langle R_{0}^{2}\right\rangle},
$$

and the chain Helmholtz free energy is given by

$$
\begin{aligned}
A_{\text {chain }}(R) & =U_{\text {chain }}(R)-T S_{\text {chain }}(R) \\
& =-k_{B} T \ln C+k_{B} T \frac{3 R^{2}}{2\left\langle R_{0}^{2}\right\rangle},
\end{aligned}
$$

where $U_{\text {chain }}(R)$ is the chain internal energy (equal to zero for the ideal chain). Equation (9) can be rewritten as

$$
A_{\text {chain }}(R)+C^{\prime}=k_{B} T \frac{3 R^{2}}{2\left\langle R_{0}^{2}\right\rangle},
$$

where $C^{\prime}=k_{B} T \ln C$, and is a constant. By comparing Eq. (10) to the equation for the elastic energy of a classical spring of length $R, A_{\text {spring }}=k_{\text {spring }} R^{2} / 2$, where $k_{\text {spring }}$ is the classical spring constant, we can recover the equivalent "spring constant" of the ideal chain, ${ }^{60} k_{\text {chain }}=3 k_{B} T /\left\langle R_{0}^{2}\right\rangle$. The chain Helmholtz free energy can then be written as

$$
A_{\text {chain }}(R)+C^{\prime}=\frac{k_{\text {chain }}}{2} R^{2} .
$$

If the network is assumed to consist of $N_{c}$ similar, parallel, and indistinguishable chains which exert equal restoring forces, then the total (elastic) free energy of the athermal network is simply the sum of the individual chain energies,

$$
A_{\text {affine }}^{\text {net }}+C^{\prime \prime}=\frac{3 N_{c} k_{B} T\left\langle R^{2}\right\rangle}{2\left\langle R_{0}^{2}\right\rangle},
$$

where $\left\langle R^{2}\right\rangle$ is the mean-squared end-to-end distance of the network chains and $C^{\prime \prime}$ is a constant.

When the network is deformed, the chain vectors are assumed to transform affinely with macroscopic deformation, i.e.,

$$
\left\langle R^{2}\right\rangle=\lambda^{2}\left\langle R_{0}^{2}\right\rangle,
$$

where $\left\langle R^{2}\right\rangle$ is the mean of the squared end-to-end vectors of the deformed network chains, averaged over all the chains. The assumption underlying Eq. (13) is that the chain vector transformation with deformation is independent of the presence of its neighbors. The free energy of the deformed athermal network in the affine model can be obtained by substituting Eq. (13) in Eq. (12),

$$
A_{\mathrm{affine}}^{\mathrm{net}}+C^{\prime \prime}=\frac{3 N_{c} k_{B} T \lambda^{2}}{2} \text {. }
$$

In addition to the free energy resulting from the loss of chain configurational entropy, Flory ${ }^{61}$ suggested that another free energy term should be added to Eq. (14) which accounts for the change in entropy of the crosslinks due to the increased volume available to them in an expanded network. The total network free energy is then ${ }^{61}$

$$
A_{\text {affine }}^{\text {net }}(\lambda)+C^{\prime \prime}=\frac{3 N_{c} k_{B} T \lambda^{2}}{2}-N_{f} k_{B} T \log \left(\lambda^{3}\right),
$$

where $N_{f}$ is the number of crosslinks of type $f(f=3,4$, etc.). This equation can be rewritten in terms of the network packing fraction using the relation [cf. Eq. (5)], $\lambda^{3}=\eta_{0} / \eta$,

$$
A_{\text {affine }}^{\text {net }}(\eta)+C^{\prime \prime}=\frac{3 N_{c} k_{B} T}{2}\left(\frac{\eta_{0}}{\eta}\right)^{\frac{2}{3}}-N_{f} k_{B} T \log \left(\frac{\eta_{0}}{\eta}\right) .
$$

The pressure of the deformed network in the affine model is given by

$P_{\text {affine }}^{\text {net }}(\eta)=-\left.\frac{\partial A_{\text {affine }}^{\text {net }}}{\partial V}\right|_{T}=-\frac{N_{c} k_{B} T}{V_{0}}\left(\frac{\eta}{\eta_{0}}\right)^{1 / 3}+\frac{N_{f} k_{B} T}{V_{0}}\left(\frac{\eta}{\eta_{0}}\right)$.

We can express $N_{c} / V_{0}$ and $N_{f} / V_{0}$ in the pressure equation above in terms of the network packing fraction using the packing fraction definition, $\eta_{0}=\pi\left(N_{c} n\right) \sigma^{3} / 6 V_{0}$, and the relation, $N_{f}=2 N_{c} / f$, between the number of crosslinks, $N_{f}$, and the number of chains, $N_{c}$, in a perfect network. Then, $\left.P_{\text {affine }}^{\text {net }} \eta\right)$ can be rewritten as 


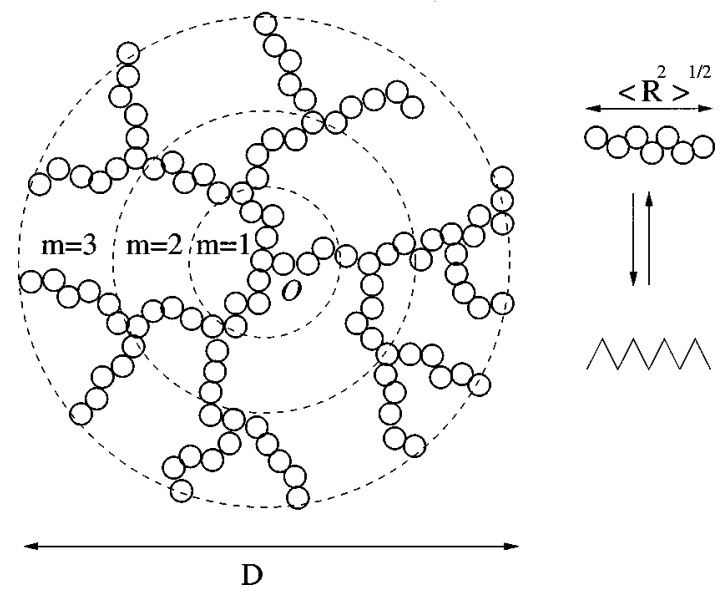

FIG. 1. Model of a single dendrimer in the network.

$$
\frac{P_{\mathrm{affine}}^{\mathrm{net}}(\eta) \sigma^{3}}{k_{B} T}=\frac{6 \eta_{0}}{\pi n}\left[-\left(\frac{\eta}{\eta_{0}}\right)^{1 / 3}+\frac{2}{f}\left(\frac{\eta}{\eta_{0}}\right)\right] .
$$

The restoring force per unit initial area (stress) is given by

$$
\begin{aligned}
& \tau=\left.\frac{1}{L_{0}^{2}} \frac{\partial A_{\text {affine }}^{\text {net }}}{\partial L}\right|_{T}=\frac{3 N_{c} k_{B} T \lambda}{V_{0}}-\frac{3 N_{f} k_{B} T}{\lambda V_{0}} \\
& \frac{\tau \sigma^{3}}{k_{B} T}=\frac{6 \eta_{0}}{\pi n}\left[3\left(\frac{\eta_{0}}{\eta}\right)^{1 / 3}-\frac{6}{f}\left(\frac{\eta}{\eta_{0}}\right)^{1 / 3}\right] .
\end{aligned}
$$

A bridge between the affine and phantom chain models, and the dendrimer-network model proposed in this article, is provided by the work of Duiser and Staverman ${ }^{58}$ and Graessley $^{59}$ who both modeled crosslinked polymers as micronetworks of ideal chains. These micronetworks are ensembles of small networks having mobile inner junctions (central junctions) and fixed outer junctions. A statistical mechanical approach based on the affine and the phantom models is employed in conjunction with the affine assumption [Eq. (13)] to obtain the restoring force per unit initial area to be

$$
\frac{\tau}{k_{B} T}=\frac{(f-2)}{f} \frac{3 N_{c} \lambda}{V_{0}}=\frac{(f-2)}{f} \frac{18 \eta_{0}}{\pi n \sigma^{3}}\left(\frac{\eta_{0}}{\eta}\right)^{1 / 3},
$$

which has the same form as the first term of Eq. (20) with the exception of the prefactor, $(f-2) / f$.

We now describe our approach to calculating network properties. Following James and Guth, we assume that the network free energy, and hence the pressure, is the sum of an elastic contribution and a liquid-like contribution. The elastic contribution is calculated by modeling the network as a set of interpenetrating dendritic structures, each of whose branches is either an ideal, gaussian chain, or a non-Gaussian chain (i.e., a chain having a non-Gaussian end-to-end vector probability distribution). Figure 1 is an example of a single such dendritic structure. It consists of concentric layers of branches, where each branch represents a network chain of end-to-end distance $\left\langle R^{2}\right\rangle^{1 / 2}$ (as shown by the right hand side of Fig. 1). The total number of concentric layers is called the rank of the dendrimer. The first (innermost) layer of the dendrimer contains $f$ branches or chains, the second layer contains $f(f-1)$ branches or chains, the third layer consists of $f(f-1)^{2}$ branches or chains, and so on. The total number of chains in a dendrimer of rank $m$ is given by

$$
\begin{aligned}
n_{\mathrm{den}} & =f+f(f-1)+f(f-1)^{2}+\cdots+f(f-1)^{(m-1)} \\
& =f\left[\frac{(f-1)^{m}-1}{(f-2)}\right],
\end{aligned}
$$

where $f$ is the functionality of the dendrimer $(f=3,4 \ldots)$. The spatial dimension or diameter of the dendrimer of rank $m$ is denoted by $D$, and is given by the relation, $D^{2}$ $=2 m\left\langle R^{2}\right\rangle$, i.e., $D^{2}$ is the square of the distance covered by a random walk beginning at any point on the circumference of the dendrimer, passing through each layer and through the origin of the dendrimer, and ending at another point on its circumference.

The elastic free energy contribution for our dendrimer model is first calculated for the case in which the network chains are modeled as ideal Gaussian chains (the Gaussian dendrimer network). The same approach is subsequently extended to the case in which the network chains are modeled as non-Gaussian chains (the non-Gaussian dendrimer network).

We now derive the free energy for a Gaussian dendrimer network. The free energy of a network of interpenetrated dendrimers can be obtained by replacing each dendrimer (composed of $n_{\text {den }}$ ideal gaussian chains of "spring constant," $k_{\text {chain }}=3 k_{B} T /\left\langle R_{0}^{2}\right\rangle$ ) by a single equivalent chain, of spring constant, $k_{\text {dendrimer }}$. Using a simple spring-resistor analogy, $k_{\text {dendrimer }}$ can be obtained by treating the dendrimer as a set of springs arranged in series and in parallel conformations, as described in Appendix A. For a dendrimer of rank $m$, containing $n_{\text {den }}$ chains, the spring constant is given by

$$
\begin{aligned}
k_{\text {dendrimer }} & =k_{\text {chain }} f(f-2)\left[\frac{(f-1)^{(m-1)}}{(f-1)^{m}-1}\right] \\
& =\frac{3 k_{B} T}{\left\langle R_{0}^{2}\right\rangle} f(f-2)\left[\frac{(f-1)^{(m-1)}}{(f-1)^{m}-1}\right] .
\end{aligned}
$$

The Helmholtz free energy of the dendrimer, $A_{\text {dendrimer }}(\eta)$, can then be obtained in a form similar to Eq. (10) by replacing the term $k_{\text {chain }}$ in Eq. (11) with $k_{\text {dendrimer }}$, and by replacing the squared end-to-end distance, $R^{2}$, of the chain, with the squared spatial dimension, $D^{2}=2 m\left\langle R^{2}\right\rangle$, of the equivalent dendrimer spring. The term, $\left\langle R^{2}\right\rangle$, represents the mean-squared end-to-end distance of a constituent chain within the dendrimer, averaged over all the $n_{\text {den }}$ dendrimer chains. When the network deforms we assume that the dendrimer also deforms affinely, that is, $D^{2}=\lambda^{2} D_{0}^{2}$, where $D_{0}^{2}$ $=2 m\left\langle R_{0}^{2}\right\rangle$ denotes the mean-squared spatial dimension of 
the undeformed dendrimer. The Helmholtz free energy of the deformed dendrimer of rank, $m$, can be written as

$$
\begin{aligned}
& A_{\text {dendrimer }}(\eta)+C^{\prime} \\
& =\frac{k_{\text {dendrimer }}}{2} D^{2} \\
& =\frac{k_{\text {dendrimer }}}{2} D_{0}^{2} \lambda^{2} \\
& =\frac{3 k_{B} T}{2\left\langle R_{0}^{2}\right\rangle} f(f-2)\left[\frac{(f-1)^{(m-1)}}{(f-1)^{m}-1}\right] 2 m\left\langle R_{0}^{2}\right\rangle \lambda^{2} \\
& =3 k_{B} T m f(f-2)\left[\frac{(f-1)^{(m-1)}}{(f-1)^{m}-1}\right]\left(\frac{\eta_{0}}{\eta}\right)^{2 / 3} .
\end{aligned}
$$

Thus far we have focused on the free energy of a single dendritic structure in the network. Since the network consists of identical dendritic structures, the free energy of the network is simply taken to be the sum of the free energies of these individual dendritic structures. The free energy of a network composed of $N_{D}$ dendrimers is then given by

$$
\begin{aligned}
A_{\text {dendrimer }}^{\text {net }}(\eta)+C^{\prime \prime}= & N_{D}\left[A_{\text {dendrimer }}(\eta)+C^{\prime}\right] \\
= & N_{D} 3 k_{B} T m f(f-2)\left[\frac{(f-1)^{(m-1)}}{(f-1)^{m}-1}\right] \\
& \times\left(\frac{\eta_{0}}{\eta}\right)^{2 / 3}
\end{aligned}
$$

where $N_{D}$ is obtained from the undeformed network packing fraction, $\eta_{0}$, using the relation,

$$
\eta_{0} \equiv \pi N_{D} n_{\mathrm{den}} n \sigma^{3} / 6 V_{0}
$$

The quantity, $n_{\text {den }}$ is given by Eq. (21). The reduced pressure of a network composed of dendrimers of rank $m$ is then given by

$$
\begin{aligned}
\frac{P_{\text {dendrimer }}^{\text {net }}(\eta) \sigma^{3}}{k_{B} T} & =-\frac{\partial A_{\text {dendrimer }}^{\text {net }}(\eta)}{\partial V} \frac{\sigma^{3}}{k_{B} T} \\
= & -\frac{12 \eta_{0}}{\pi n} m(f-2)^{2} \\
& \times\left[\frac{(f-1)^{(m-1)}}{\left((f-1)^{m}-1\right)^{2}}\right]\left(\frac{\eta}{\eta_{0}}\right)^{1 / 3} .
\end{aligned}
$$

Next, we derive the free energy for a non-Gaussian dendrimer network. Although the probability distribution for the end-to-end distances of the network chains is adequately described by the Gaussian distribution [Eq. (7)] for small deformations, at large deformations approaching the maximum extensibility of the chains, this distribution is expected to show significant deviations from the Gaussian form. Kuhn and Grun $^{43}$ suggested the following non-Gaussian form for the probability distribution of the chains, based on the concept that a chain has finite maximum extensibility:

$$
\begin{aligned}
p_{\text {chain }}(\mathbf{R})= & C_{1} \exp \left\{-n\left[\frac{3}{2}\left(\frac{R}{n \sigma}\right)^{2}+\frac{9}{20}\left(\frac{R}{n \sigma}\right)^{4}\right.\right. \\
& \left.\left.+\frac{99}{350}\left(\frac{R}{n \sigma}\right)^{6}+\cdots\right]\right\},
\end{aligned}
$$

where $C_{1}$ is a constant, and $n$ is the chain length. As in the case of the Gaussian distribution, this non-Gaussian distribution is also based on the assumption that the chains have no excluded volume (i.e., that the chains can pass through each other). The non-Gaussian distribution reduces to the Gaussian form [Eq. (7)] when the higher order terms, $(R / n \sigma)^{4}$ and $(R / n \sigma)^{6}$, in the above equation are small enough to be neglected, and when $n \sigma^{2}$ is taken to be equal to $\left\langle R_{0}^{2}\right\rangle$ (the relation between the end-to-end distance of an ideal, Gaussian chain and its chain length). Deviations in $p(R)$ from the Gaussian are about $8 \%$ when the end-to-end distance of the chain is 0.5 times its fully extended length, and about $20 \%$ when the end-to-end distance of the chain is 0.7 times its fully extended length.

We now outline the steps by which the network free energy can be obtained for the dendrimer-network model with a non-Gaussian chain vector distribution. The general method is based on the approach described by Treloar ${ }^{44}$ in which many of the assumptions of the affine model (with Gaussian chains) are applied to the non-Gaussian chain case. Our method for the non-Gaussian dendrimer network is similar to our approach in the Gaussian dendrimer-network case, and involves making similar assumptions, including the neglect of the chain excluded volume, and the use of the affine assumption. We begin by obtaining the free energy of a single non-Gaussian chain in analogy with the method described earlier for the single Gaussian chain [cf. Eqs. (7)(11)]

$$
\begin{aligned}
A_{\text {chain }}(R)= & -T S_{\text {chain }}(R) \\
= & -k_{B} T \log p_{\text {chain }}(R) \\
= & -k_{B} T \log C_{1}+k_{B} T n\left[\frac{3}{2}\left(\frac{R}{n \sigma}\right)^{2}+\frac{9}{20}\left(\frac{R}{n \sigma}\right)^{4}\right. \\
& \left.+\frac{99}{350}\left(\frac{R}{n \sigma}\right)^{6}+\cdots\right] .
\end{aligned}
$$

By comparing this to the equation for the elastic free energy of a classical spring, the spring constant for the nonGaussian chain can be written as

$k_{\text {chain }}=k_{B} \operatorname{Tn}\left[\frac{3}{(n \sigma)^{2}}+\frac{9}{10} \frac{R^{2}}{(n \sigma)^{4}}+\frac{99}{175} \frac{R^{4}}{(n \sigma)^{6}}+\cdots\right]$.

Thus the spring constant of a non-Gaussian chain depends on the chain end-to-end distance, unlike that of a Gaussian chain.

The calculation of the elastic free energy of a network of non-Gaussian chains is based on the assumption ${ }^{44}$ that the non-Gaussian probability distribution [Eq. (27)] can be treated as the product of its components along the $x, y$, and $z$ axes, i.e., $p_{\text {chain }}(R)=p_{\text {chain }}(X) p_{\text {chain }}(Y) p_{\text {chain }}(Z)$. This assumption is strictly valid for the Gaussian case [cf. Eq. (7)] but is commonly extended to the non-Gaussian case. ${ }^{44}$ In this instance, this assumption allows us to calculate the free en- 
ergy of the non-Gaussian dendrimer network in the same way as for the Gaussian dendrimer network. The Helmholtz free energy of the non-Gaussian dendrimer network is obtained by evaluating the free energy of a single non-Gaussian dendrimer, $A_{\text {dendrimer }}(\eta)$, in analogy with the treatment of the Gaussian dendrimer network in Eq. (23). To do this, the effective spring constant for each dendritic structure is again taken to be $k_{\text {dendrimer }}=k_{\text {chain }} f(f-2)\left[(f-1)^{(m-1)} /(f-1)^{m}\right.$ $-1]$, as in Eq. (22). Again, as in the Gaussian dendrimernetwork case, we average the chain dimensions over all the network chains, i.e., we replace the chain end-to-end distance $R^{2}$ with $\left\langle R^{2}\right\rangle$, and $R^{4}$ with $\left\langle R^{4}\right\rangle$. Using the affine assumption, $\left\langle R^{2}\right\rangle=\lambda^{2}\left\langle R_{0}^{2}\right\rangle$, and assuming further that $\left\langle R^{4}\right\rangle$ $=\lambda^{4}\left\langle R_{0}^{4}\right\rangle$, the Helmholtz free energy of a deformed nonGaussian dendrimer can be written in a form analogous to Eq. (23) as

$$
\begin{aligned}
A_{\text {dendrimer }}(\eta)+C_{1}^{\prime}= & \frac{k_{\text {dendrimer }}}{2} D^{2} \\
= & \frac{3 k_{B} T}{2} f(f-2)\left[\frac{(f-1)^{(m-1)}}{(f-1)^{m}-1}\right] \\
& \times\left[\frac{1}{n \sigma^{2}}+\frac{3}{10} \frac{\left\langle R_{0}^{2}\right\rangle \lambda^{2}}{n\left(n \sigma^{2}\right)^{2}}\right. \\
& \left.+\frac{33}{175} \frac{\left\langle R_{0}^{4}\right\rangle \lambda^{4}}{n^{2}\left(n \sigma^{2}\right)^{3}}+\cdots\right] 2 m\left\langle R_{0}^{2}\right\rangle \lambda^{2} .
\end{aligned}
$$

We use the relation, $\left\langle R_{0}^{2}\right\rangle \equiv n \sigma^{2}$ (since the undeformed network chains are Gaussian), the approximation, $\left\langle R_{0}^{4}\right\rangle \approx\left\langle R_{0}^{2}\right\rangle^{2}$ $\equiv\left(n \sigma^{2}\right)^{2}$, to recast the equation in the following form:

$$
\begin{aligned}
A_{\text {dendrimer }}(\eta)+C_{1}^{\prime}= & 3 k_{B} T m f(f-2)\left[\frac{(f-1)^{(m-1)}}{(f-1)^{m}-1}\right] \\
& \times\left[\lambda^{2}+\frac{3}{10} \frac{\lambda^{4}}{n}+\frac{33}{175} \frac{\lambda^{6}}{n^{2}} \cdots\right] .
\end{aligned}
$$

The free energy of the non-Gaussian dendrimer network is obtained by summing the free energies of $N_{D}$ dendrimers [as done in Eq. (24)], and is given by

$$
A_{\text {dendrimer }}^{\text {net }}(\eta)+C_{1}^{\prime \prime}=N_{D}\left(A_{\text {dendrimer }}(\eta)+C_{1}^{\prime}\right),
$$

where $N_{D}$ is related to $\eta_{0}$ as shown in Eq. (25). The reduced pressure of the network composed of non-Gaussian dendrimers of rank $m$ is then

$$
\begin{aligned}
\frac{P_{\text {dendrimer }}^{\text {net }}(\eta) \sigma^{3}}{k_{B} T}= & -\frac{\partial A_{\text {dendrimer }}^{\text {net }}}{\partial V} \frac{\sigma^{3}}{k_{B} T} \\
= & -\frac{6 \eta_{0}}{\pi n} m(f-2)^{2}\left[\frac{(f-1)^{(m-1)}}{\left((f-1)^{m}-1\right)^{2}}\right] \\
& \times\left[2\left(\frac{\eta}{\eta_{0}}\right)^{1 / 3}+\frac{6}{5 n}\left(\frac{\eta}{\eta_{0}}\right)^{-1 / 3}\right. \\
& \left.+\frac{198}{175 n^{2}}\left(\frac{\eta}{\eta_{0}}\right)^{-1}+\cdots\right] .
\end{aligned}
$$

This equation reduces to the Gaussian dendrimer-network pressure equation [Eq. (26)] when the second and third terms in the square bracket on the right side are negligible, i.e., at small deformations of the network.

Finally we calculate the liquid-like contribution to the free energy of a network. Since our networks are athermal, this term arises from the intersegment repulsions between the network monomers. It can be obtained by using an approach based on the GFD theory of Dickman, Honnell, and Hall, ${ }^{40-42}$ which has been successfully used to evaluate the thermodynamic properties of a variety of hard-chain systems. We begin by briefly reviewing some pertinent results of the GFD theory for uncrosslinked, linear hard-sphere chains.

The GFD prediction for the compressibility factor of a fluid of hard-chain $n$-mers is given by

$$
\begin{aligned}
Z_{\mathrm{GFD}}(\eta)= & {\left[\frac{v_{e}(n)-v_{e}(1)}{v_{e}(2)-v_{e}(1)}\right] Z_{2}(\eta) } \\
& -\left[\frac{v_{e}(n)-v_{e}(2)}{v_{e}(2)-v_{e}(1)}\right] Z_{1}(\eta),
\end{aligned}
$$

where $Z_{1}(\eta)$ and $Z_{2}(\eta)$ are the compressibility factors of fluids composed of hard sphere monomers and dimers, respectively, at the same packing fraction as the $n$-mer fluid, $v_{e}(1), v_{e}(2)$ are the monomer and dimer excluded volumes, and $v_{e}(n)$ is the volume excluded by an $n$-mer to a monomer. The excluded volumes of a monomer, dimer, and trimer can be evaluated exactly, and are given by $v_{e}(1)$ $=(4 \pi / 3) \sigma^{3}, \quad v_{e}(2)=(9 \pi / 4) \sigma^{3}$, and $v_{e}(3)=9.82605 \sigma^{3}$. The excluded volume of an $n$-mer linear chain is difficult to evaluate exactly; the following approximate form was used by Dickman and Hall ${ }^{40}$

$$
v_{e}(n)=v_{e}(3)+(n-3)\left[v_{e}(3)-v_{e}(2)\right] .
$$

The compressibility factor for the monomer fluid, $Z_{1}(\eta)$, is obtained from the Carnahan-Starling equation of state ${ }^{62}$

$$
Z_{1}(\eta)=\frac{1+\eta+\eta^{2}-\eta^{3}}{(1-\eta)^{3}}
$$

and the compressibility factor for the dimer fluid, $Z_{2}(\eta)$, is obtained from the Tildesley-Streett equation of state ${ }^{63}$

$$
Z_{2}(\eta)=\frac{1+2.45696 \eta+4.10386 \eta^{2}-3.75503 \eta^{3}}{(1-\eta)^{3}}
$$

Thus, from the GFD equations of state, we can obtain the pressure of a chain fluid at any packing fraction based on knowledge of the hard-monomer and dimer compressibility factors and the excluded volume of the chain molecule.

To evaluate the liquid-like contribution to the network pressure, $P_{\text {liq }}^{\text {net }}$, we postulate that $P_{\text {liq }}^{\text {net }}$ is equal to the pressure of a reference polymer having the same excluded volume as the network. For the dendrimer-network model described earlier in Sec. III, the reference polymer is simply a fluid of $f$-functional dendrimers of rank $m$. To calculate the compressibility factor of this dendrimer fluid using the GFD equations, we need only evaluate the excluded volume of a single dendrimer. To do so, we note that a dendrimer is es- 


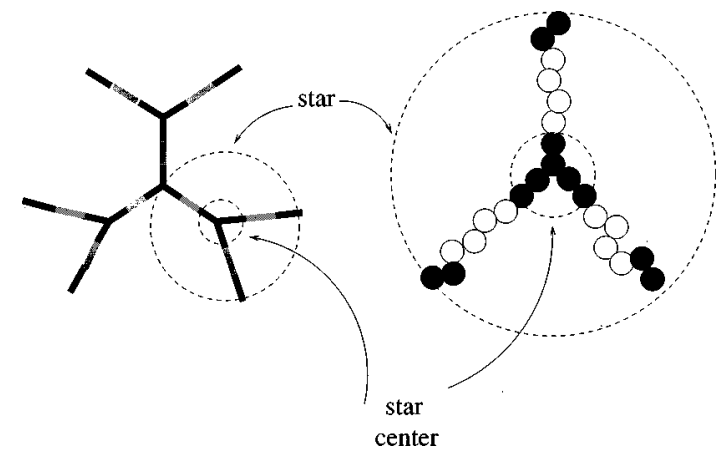

FIG. 2. (a) Portion of a trifunctional dendrimer consisting of three stars. The dendrimer shown contains four star centers (dark lines) with connecting chains (light lines). (b) One of the constituent stars is enlarged to show that the star center consists of $2 f$ segments (dark beads) and that the connecting chains consist of $(n-4)$ segments each (light beads).

sentially a set of connected $f$-functional star molecules, as illustrated in Fig. 2. To calculate the excluded volume of the dendrimer we need to evaluate the total excluded volume of this set of connected star molecules. In this context it is useful to consider the expression developed by Yethiraj and Hall $^{64}$ for the excluded volume of a star in a fluid of unconnected star molecules

$v_{e}\left(n_{\text {star }}, f\right)=v_{\text {center }}(f)+f\left(\frac{n_{\text {star }}-1}{f}-2\right)\left[v_{e}(3)-v_{e}(2)\right]$,

where $n_{\text {star }}$ is the number of segments in the star, and $v_{\text {center }}(f)$ is the excluded volume of the star center. In Eq. (39), the star center consists of $2 f+1$ segments - two segments from each arm of the star and one central bead. The excluded volume of the star center is not obtained analytically, but is calculated from a Monte Carlo simulation in which a monomer is inserted successively at many randomly chosen points in a box containing random configurations of the star center (Alejandre and Chapela, ${ }^{65}$ Yethiraj and Hall $^{64}$.

We can employ an equation similar to Eq. (38) to calculate the excluded volume of a dendrimer of rank $m$. To do so we subdivide the dendrimer into a set of star centers connected by arms which are simply linear chains. Figure 2 shows a portion of a dendrimer consisting of three connected star molecules (the left side of Fig. 2) and a single star molecule enlarged to show the constituent beads (the right side of Fig. 2). For each star molecule on the left side, the dark lines denote the star centers and the lighter lines denote the chains connecting them, while for the enlarged star molecule on the right side, the dark beads denote the star centers and the light beads denote the chain segments connecting them. Each star center is composed of $2 f$ segments, i.e., two segments from each arm of the star (there is no central bead since the chains are endlinked). Each connecting chain is composed of $(n-4)$ segments, where $n$ is the length of the network chains. The chains in the last layer of the network, being connected to star centers only at one end, are composed of $(n-2)$ segments each. A dendrimer of rank $m$ will contain $1+f\left[(f-1)^{m-1}-1\right] /(f-2)$ star centers and $n_{\text {den }}$ chains [cf. Eq. (21)] of which $f(f-1)^{(m-1)}$ chains are in the last layer of the network. The total dendrimer excluded volume will be

$$
\begin{aligned}
v_{\text {dendrimer }}(n, m, f)= & {\left[1+f \frac{(f-1)^{(m-1)}-1}{(f-2)}\right] v_{\text {center }}(f) } \\
& +(n-4)\left[\frac{f(f-1)^{m}-1}{(f-2)}\right] \\
& \times\left[v_{e}(3)-v_{e}(2)\right]+2 f(f-1)^{(m-1)} \\
& \times\left[v_{e}(3)-v_{e}(2)\right] .
\end{aligned}
$$

The excluded volumes, $v_{\text {center }}(f)$, of the trifunctional and tetrafunctional star centers are calculated using the Monte Carlo algorithm mentioned above. ${ }^{64,65}$ For a trifunctional network $(f=3)$, the star center contains six segments and $v_{\text {center }}(3)=18.01 \pm 0.05$; for a tetrafunctional network $(f$ $=4)$, the star center contains eight segments and $v_{\text {center }}(4)$ $=23.06 \pm 0.03$.

The compressibility factor of the fluid of dendrimers, each of excluded volume, $v_{\text {dendrimer }}(n, m, f)$, and rank, $m$, can then be calculated from

$$
\begin{aligned}
Z_{\mathrm{GFD}}^{\mathrm{net}}(\eta, f)= & {\left[\frac{v_{\text {dendrimer }}(n, m, f)-v_{e}(1)}{v_{e}(2)-v_{e}(1)}\right] Z_{2}(\eta) } \\
& -\left[\frac{v_{\text {dendrimer }}(n, m, f)-v_{e}(2)}{v_{e}(2)-v_{e}(1)}\right] Z_{1}(\eta) .
\end{aligned}
$$

The liquid-like contribution to the network pressure is obtained from the relation, $P_{\mathrm{GFD}}^{\text {net }}(\eta, f) / k_{B} T \equiv Z_{\mathrm{GFD}}^{\text {net }} N_{D} / V$ $=Z_{\mathrm{GFD}}^{\text {net }}(\eta, f) 6 \eta / \pi \sigma^{3}\left(n_{\mathrm{den}} n\right)$ where $\left(n_{\mathrm{den}} n\right)$ is the number of segments in a dendrimer, and $n_{\text {den }}$ is obtained from Eq. (21). The reduced liquid-like contribution to the network pressure is given by

$$
\frac{P_{\mathrm{GFD}}^{\mathrm{net}} \sigma^{3}}{k_{B} T}=Z_{\mathrm{GFD}}^{\mathrm{net}}(\eta, f) \frac{6 \eta}{\pi n}\left\{\frac{(f-2)}{f\left((f-1)^{m}-1\right)}\right\} .
$$

The total reduced pressure of the network is the sum of the liquid-like contribution [Eq. (41)] and the elastic contribution. The elastic contribution to the pressure has been calculated for the cases, (1) a dendrimer network of Gaussian chains [Eq. (26)], and (2) a dendrimer network of nonGaussian chains (chains that are Gaussian at low deformations and non-Gaussian at large deformations) [Eq. (33)]. The total reduced network pressure, $P^{\text {net }} \sigma^{3} / k_{B} T$ $=P_{\mathrm{GFD}}^{\text {net }} \sigma^{3} / k_{B} T+P_{\text {dendrimer }}^{\text {net }} \sigma^{3} / k_{B} T$ is given for the Gaussian dendrimer network by

$$
\begin{aligned}
\frac{P^{\mathrm{net}} \sigma^{3}}{k_{B} T}= & Z_{\mathrm{GFD}}^{\mathrm{net}}(\eta, f) \frac{6 \eta}{\pi n}\left\{\frac{(f-2)}{f\left[(f-1)^{m}-1\right]}\right\} \\
& -\frac{12 \eta_{0}}{\pi n} m(f-2)^{2}\left\{\frac{(f-1)^{(m-1)}}{\left[(f-1)^{m}-1\right]^{2}}\right\}\left(\frac{\eta}{\eta_{0}}\right)^{1 / 3},
\end{aligned}
$$

and for the non-Gaussian dendrimer network by 
TABLE III. Simulation results for the reduced pressure, $P^{*}=P \sigma^{3} / k_{B} T$, as a function of packing fraction, $\eta$, for trifunctional networks at chain lengths, $n=20,35,50$, and 100 .

\begin{tabular}{ccccc}
\hline \hline$\eta$ & 20 & 35 & 50 & 100 \\
\hline 0.45 & 5.129 & 5.149 & 5.155 & 5.166 \\
0.40 & 3.021 & 3.042 & 3.048 & $\ldots$ \\
0.35 & 1.736 & 1.755 & 1.758 & 1.769 \\
0.30 & 0.959 & 0.973 & 0.975 & $\ldots$ \\
0.25 & 0.495 & 0.507 & 0.509 & 0.516 \\
0.15 & 0.087 & 0.095 & 0.094 & 0.099 \\
0.10 & 0.018 & 0.025 & 0.024 & 0.028 \\
0.05 & -0.0065 & -0.00098 & -0.0017 & -0.0014 \\
0.03 & -0.0107 & -0.0045 & -0.0049 & -0.0017 \\
0.01 & -0.0224 & -0.0074 & -0.0075 & -0.0027 \\
0.005 & $\cdots$ & -0.012 & -0.0118 & $\ldots$ \\
0.0028 & $\cdots$ & $\ldots$ & $\cdots$ & -0.0028 \\
\hline \hline
\end{tabular}

$$
\begin{aligned}
\frac{P^{\mathrm{net}} \sigma^{3}}{k_{B} T}= & Z_{\mathrm{GFD}}^{\mathrm{net}}(\eta, f) \frac{6 \eta}{\pi n}\left\{\frac{(f-2)}{f\left[(f-1)^{m}-1\right]}\right\} \\
& -\frac{6 \eta_{0}}{\pi n} m(f-2)^{2}\left\{\frac{(f-1)^{(m-1)}}{\left[(f-1)^{m}-1\right]^{2}}\right\}\left[2\left(\frac{\eta}{\eta_{0}}\right)^{1 / 3}\right. \\
& \left.+\frac{6}{5 n}\left(\frac{\eta}{\eta_{0}}\right)^{-1 / 3}+\frac{198}{175 n}\left(\frac{\eta}{\eta_{0}}\right)^{-1}+\cdots\right]
\end{aligned}
$$

\section{RESULTS AND DISCUSSION}

In this section, we present our simulation results for the pressure-volume properties of trifunctional and tetrafunctional networks. The simulation results for the network pressure are compared with the predictions of the constrained junction model, ${ }^{5,6}$ the Obhukov, Rubinstein, and Colby model, ${ }^{53}$ the affine model, and our Gaussian and nonGaussian dendrimer-network models. Finally we compare our results for the pressure and chain dimensions for the trifunctional networks with those for tetrafunctional networks, and with those for uncrosslinked chain systems.

Table III shows the simulation results for the reduced pressure, $P^{*}=P \sigma^{3} / k_{B} T$, for trifunctional networks of chain length $20,35,50$, and 100 , over a packing fraction range of $0.45-\sim 0.003$. Each value of the pressure shown in Table III is calculated from simulation runs of between 500 million and a billion collisions (depending on the system size). It was not possible to repeat these runs for each of the data points because of the large system sizes in this study (between 1800 and 9000 segments), and because long simulation runs are required for network systems. For selected systems three or more runs were performed in order to estimate the standard deviation for the data. From our sampling of the data, the error associated with the pressure was found to be less than $0.1 \%$.

Figure 3 shows our simulation results for the reduced pressure of the trifunctional networks as a function of packing fraction. Since the magnitudes of the pressure for the different chain length networks are similar (see Table III), the network pressure is multiplied by the chain length for clarity. We observe that the pressure shows the expected continuous increase with increasing packing fraction, which

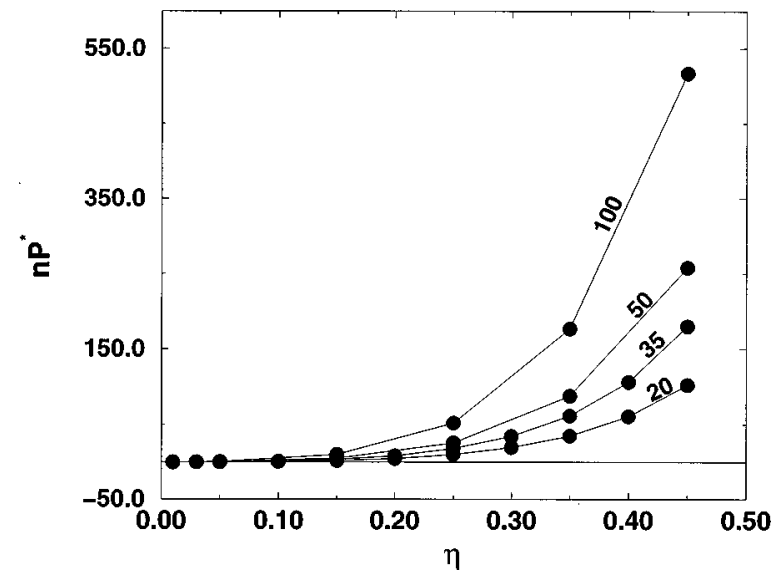

FIG. 3. Reduced pressure, $P^{*}$, multiplied by the network chain length, $n$, vs the packing fraction, $\eta$, for trifunctional networks of chain lengths, 20, 35, 50, and 100. Lines are added solely to guide the reader's eye.

would also be the case for uncrosslinked chain systems. Table III shows us that the network pressure becomes negative at very low packing fractions. Although the scale of the graph in Fig. 3 does not permit us to observe the low packing fraction region, this will be more apparent in the later figures.

The similarities and differences between the pressurevolume behavior of networks and uncrosslinked chain systems are clearly illustrated in Fig. 4, which shows simulation results for the compressibility factor of an uncrosslinked 20mer chain system, $Z_{\text {chain }}[$ cf. Eq. (6)], and the scaled compressibility factor of a 20-mer trifunctional network, $Z^{\text {net }} N_{D} / N_{c}$, as a function of packing fraction, $\eta$. The curve represents the prediction of the GFD theory ${ }^{40-42}$ for the compressibility factor of the uncrosslinked chain system. The scaled form of the network compressibility factor, $Z^{\text {net }} N_{D} / N_{c} \equiv P^{\text {net }} V / N_{c} k_{B} T=P^{*} \pi n / 6 \eta$, is used in order to facilitate comparison with the uncrosslinked chain system compressibility factor, $Z^{\text {chain }} \equiv P V / N_{c} k_{B} T=P^{*} \pi n / 6 \eta$. We see that the variation in the scaled network compressibility

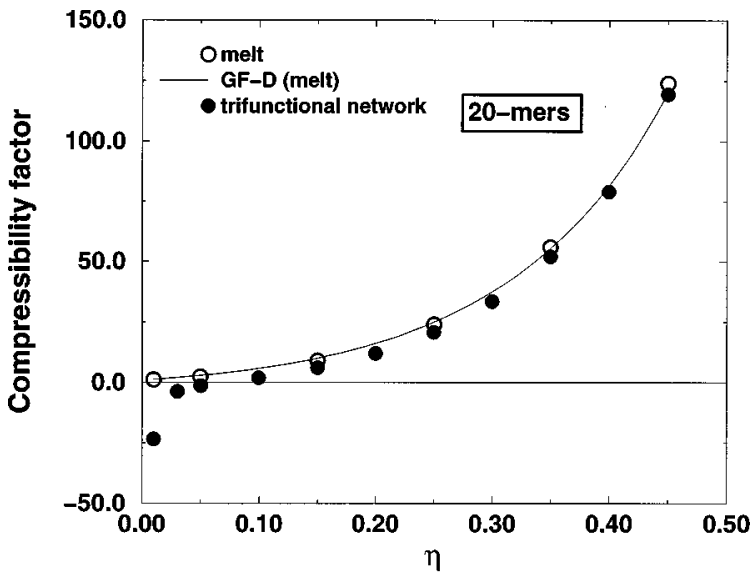

FIG. 4. Compressibility factor, $Z \equiv P^{*} n / \eta$, vs packing fraction, $\eta$, for a trifunctional network (filled circles) and an uncrosslinked chain system (open circles), both of chain length, $n=20$. The curve represents the GFD theory prediction (Refs. 40-42) for the compressibility factor of the uncrosslinked chain system. 


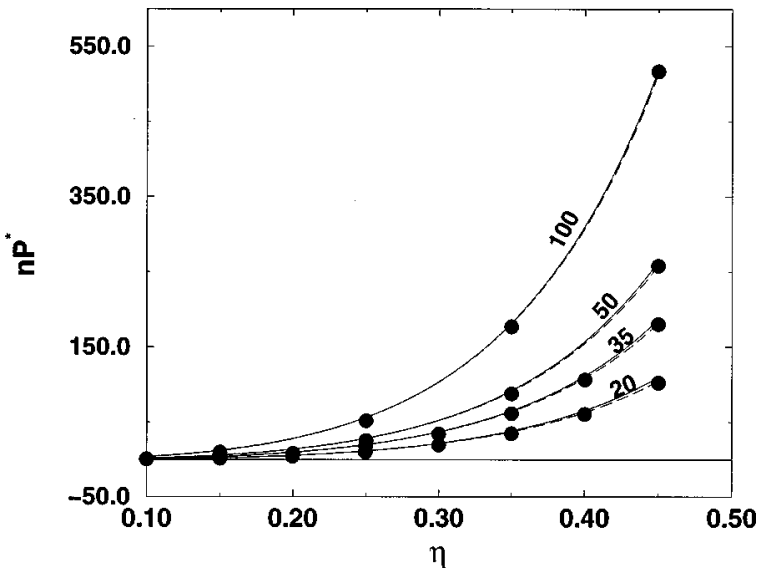

FIG. 5. Comparison of simulation results and theoretical predictions for the reduced pressure, $P^{*}$, multiplied by the network chain length, $n$, vs the packing fraction, $\eta$, for trifunctional networks of chain lengths, 20, 35, 50, and 100. Models represented are the affine model (dashed line), Gaussian dendrimer-network model, and non-Gaussian dendrimer-network model (solid lines). The predictions of all three models lie on top of each other over the entire packing fraction range shown.

factor with packing fraction is similar to that of the uncrosslinked chain system compressibility factor at high to moderate packing fractions. The compressibility factor of the network is always lower than that of the uncrosslinked chain system due to the elastic retractive force exerted by the network chains as they oppose network deformation. At very low densities $(\eta<0.1)$, the uncrosslinked chain compressibility factor tends to a value of 1 , while the network compressibility factor becomes negative. This negative region in the network compressibility factor was first observed by Escobedo and de Pablo. ${ }^{33}$ The reason for its existence is that at these low densities, the liquid-like contribution to the total pressure of the network is negligible, but the (negative) elastic contribution is relatively large.

We now compare our simulation results with the theoretical predictions for network pressure described in Sec. III. The elastic component of the pressure is obtained from the following models: (1) the affine model [Eq. (18)], (2) the Gaussian dendrimer-network model [Eq. (26)], and (3) the non-Gaussian dendrimer-network model [Eq. (33)]. The liquid-like component of the pressure is obtained from the GFD theory for chains [Eq. (34)] for the affine model, and from the GFD theory for networks [Eq. (41)] for the Gaussian and non-Gaussian dendrimer-network models. For all three models, the packing fraction of the undeformed network, $\eta_{0}$, is taken to be equal to 0.45 . For the dendrimernetwork models (Gaussian and non-Gaussian) the value of the network rank, $m$, is chosen so as to give the best agreement with simulation results. The value of $m$ is taken to be 2 for all the networks considered in this article.

Figure 5 shows the simulation results (symbols) for the reduced network pressure scaled by the chain length, $n P^{*}$, versus the packing fraction for the four trifunctional networks, $(n=20,35,50$, and 100) in the packing fraction region, $\eta=0.1-0.45$. The lines in Fig. 5 show the predictions of the affine model (dashed line), and the Gaussian dendrimer and the non-Gaussian dendrimer models (solid lines).

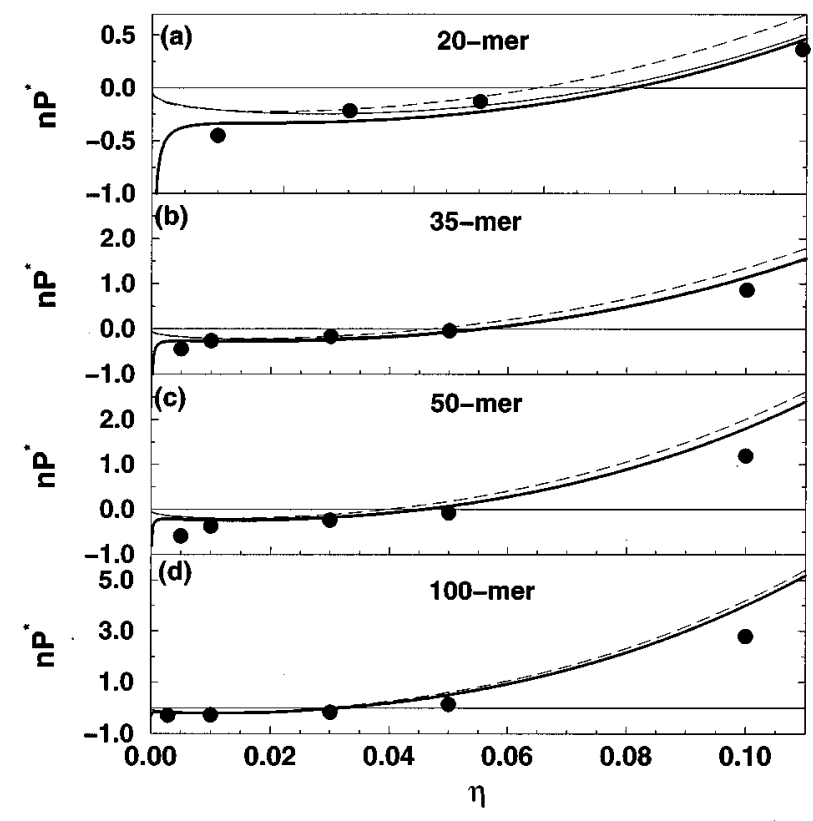

FIG. 6. Comparison of simulation results and theoretical predictions for the reduced pressure, $P^{*}$, multiplied by the chain length, $n$, vs the packing fraction, $\eta$, in the region $\eta<0.12$, for trifunctional networks of chain length: (a) 20, (b) 35, (c) 50, and (d) 100. The dashed lines represent the affine model prediction [Eqs. (18) and (34)], the solid line represents the Gaussian dendrimer-network model [Eq. (42)], and the bold line represents the non-Gaussian network model [Eq. (43)].

The predictions of the three models lie on top of each other except in the high packing fraction region for the 20-mer, 35-mer, and 50-mer networks where there is a slight discrepancy between the affine and dendrimer model predictions. The three theories are in good agreement with our simulation results for all chain lengths at high to intermediate densities.

Figure 6 shows the simulation results for the scaled network pressure, $n P^{*}$, versus packing fraction, for the four trifunctional networks in the low packing fraction region $(\eta<0.1)$ along with the prediction of the affine model (dashed line), the Gaussian dendrimer model (solid line), and the non-Gaussian dendrimer model (bold solid line). At these low packing fractions, the network pressure becomes negative, and the network models have different degrees of success. We first consider the 20-mer trifunctional network shown in Fig. 6(a). As the packing fraction decreases, a downturn in the network pressure is evidenced, which is not predicted by either the affine or the Gaussian dendrimernetwork models. In fact these two models predict a slight increase in the pressure at very low densities. However the non-Gaussian dendrimer-network model is strikingly successful in predicting the downturn in pressure, as well as in providing generally good agreement with our simulation results in the low packing fraction region. Since the theoretical basis for the non-Gaussian dendrimer-network model is the deviation in the network chain probability distribution from the Gaussian form at large network deformations, the success of this theory in predicting the network pressure at very low packing fraction (i.e., large deformations) and the failure of the affine and Gaussian dendrimer-network theories strengthen the supposition that non-Gaussian chain extension 
TABLE IV. Simulation results for the chain mean-squared end-to-end distance, $\left\langle R^{2}\right\rangle / \sigma^{2}$, at each packing fraction, $\eta$, and corresponding deformation ratio, $\lambda$, for trifunctional networks at chain lengths $n=20,35,50$, and 100 .

\begin{tabular}{lccccc}
\hline \multicolumn{1}{c}{$\eta$} & $\lambda$ & 20 & 35 & 50 & 100 \\
\hline 0.45 & 1.0 & 28.54 & 54.50 & 79.40 & 166.58 \\
0.4 & 1.04 & 31.92 & 58.14 & 87.29 & $\ldots$ \\
0.35 & 1.09 & 34.25 & 65.23 & 96.22 & 190.66 \\
0.3 & 1.14 & 37.35 & 69.10 & 103.92 & $\ldots$ \\
0.25 & 1.22 & 41.16 & 75.55 & 114.24 & 231.85 \\
0.15 & 1.44 & 52.42 & 100.67 & 156.97 & 314.42 \\
0.05 & 2.08 & 82.73 & 168.81 & 280.84 & 597.40 \\
0.03 & 2.47 & 212.94 & 361.97 & 770.87 & $\ldots$ \\
0.02 & 2.82 & 123.44 & $\ldots$ & $\ldots$ & $\ldots$ \\
0.01 & 3.56 & 181.94 & 382.46 & 667.25 & 1389.77 \\
0.005 & 4.48 & $\ldots$ & 592.41 & 1033.05 & $\ldots$ \\
0.0028 & 5.44 & $\ldots$ & $\ldots$ & $\ldots$ & 2960.19 \\
\hline \hline
\end{tabular}

effects are significant in highly deformed networks.

The non-Gaussian statistical theory of Kuhn and Grun ${ }^{43}$ [Eq. (33)] which forms the basis for our non-Gaussian dendrimer-network model, predicts that for a freely jointed chain, departures from the Gaussian statistical theory first become important when the chain extensibility, defined to be the ratio of the chain end-to-end distance, $R$, and the fully extended chain length, $n \sigma$, is between one third and one half. In light of this prediction, it is of interest to calculate the chain extensibilities at the packing fractions at which the pressure downturn shown in Fig. 6 occurs. We observe that the downturn in the pressure occurs at a packing fraction of approximately 0.01 for the 20-mer network [Fig. 6(a)], and at packing fractions of approximately 0.005 for the 35 -mer and 50-mer networks [Figs. 6(b) and 6(c) ]. The downturn in pressure does not occur at all in the 100-mer network [Fig. $6(\mathrm{~d})$ ] over the packing fraction range studied (down to $\eta$ $=0.0028$ ). To obtain the chain extensibilities at these packing fractions, we calculate the mean-squared end-to-end distances of the network chains, $\left\langle R^{2}\right\rangle / \sigma^{2}$ from our simulations, and then set the chain extensibility to be $R / n=\left\langle R^{2}\right\rangle^{1 / 2} / n$. The simulation results for the network chain mean-squared end-to-end distance $\left\langle R^{2}\right\rangle / \sigma^{2}$ are shown in Table IV. From the results in Table IV we see that for the 20-mer network, the packing fraction of 0.01 , which is where the pressure downturn occurs, corresponds to a chain extensibility of $\sim 67 \%$, i.e., the average end-to-end distance of the 20 -mer chains is approximately $67 \%$ of its fully extended length. For the 35-mer and 50-mer networks, the packing fraction of 0.005 , which is where the pressure downturn occurs, corresponds to chain extensibilities of $\sim 70 \%$ and $\sim 65 \%$, respectively. For the 100-mer network, there is no downturn in pressure and the chain extensibility is only $54 \%$ at the lowest packing fraction of 0.0028 . The chain extensibilities at which the downturns in pressure are observed are larger than those suggested by Kuhn and Grun ${ }^{43}$ (between one third and two thirds), but are in excellent agreement with the experimental results of Andrady, Llorente, and Mark, ${ }^{66}$ who observed non-Gaussian effects to begin at approximately $60 \%-$ $70 \%$ of the maximum chain extensibility.

From Fig. 6, we also see that the downturn in pressure occurs at lower packing fractions as the chain length in- creases. This is related to the inverse dependence of the chain extensibilities on chain length, i.e., at a constant packing fraction, the chain extensibility decreases with increasing chain molecular weight. This inverse dependence arises from the fact that for all ideal or real chains, the chain end-to-end distance, $R$, is related to the chain length as, $R \propto n^{a}$, where $a<1$. The chain extensibility, $R / n \sigma$, then takes the form, $R / n \sigma \propto 1 / n^{(1-a)}$; thus the extensibility decreases with increasing chain length, and a 20 -mer chain at a given packing fraction is extended to a higher fraction of its fully extended length than a 100-mer chain at the same packing fraction. Hence the 20-mer chain shows departures from Gaussian statistics at higher packing fractions (smaller deformations) than the 100-mer chain, and as a result, the Gaussian network theories will be successful over an increasing range of packing fractions as the network chain length rises. Thus for the 100-mer network, both the affine and Gaussiandendrimer models predict the network pressure fairly well even at low densities, while for the shorter chain length networks, the Gaussian theories work well only at high and intermediate densities, and fail at low densities.

The success of the non-Gaussian theory in predicting network behavior over such a wide range of packing fractions is intriguing because of two assumptions that were made in arriving at Eq. (33): the neglect of excluded volume interactions in the elastic term, and the assumption that the mean end-to-end distances of the chains deform affinely with macroscopic strain. These two assumptions are related in the sense that the neglect of excluded volume allows the affine assumption to be made, since chains that do not exclude volume could deform freely by passing through each other (being "phantoms"), and their motion would not be constrained by neighboring chains or crosslinks. Here we explore the implications of the neglect of excluded volume by comparing our simulation results to theories developed by other researchers ${ }^{5,6,53}$ in an attempt to account for excluded volume effects.

Although the complex nature of real chains makes it difficult to incorporate excluded volume interactions into the elastic free energy expression in a simple manner, several theoretical approaches have been proposed that account for specific consequences of the monomer-monomer repulsion. We focus on two such models: (1) the constrained junction model of Flory and Erman,, 5 in which the damping effects on junction motion due to the presence of the neighboring network chains are concentrated in a domain of constraint, and (2) the work of Obhukov, Rubinstein, and Colby ${ }^{53}$ in which stress-strain relations are proposed for deswollen networks based on the Pincus blob model ${ }^{67}$ for real chains. Both models assume that the crosslink mean positions (and hence the chain vectors) deform affinely with strain. The theoretical details of these models are given elsewhere, ${ }^{5,6,8,53}$ here we only show the final expressions for the network elastic free energy.

The constrained junction model (Flory and Erman, Ronca and Allegra ${ }^{68}$ ) was proposed to incorporate the damping effect of chain entanglements on the spatial fluctuations of the junctions. In this model, the free motion of the junctions in a phantom domain (i.e., in a purely phantom net- 
work) are impeded by a constraint domain that represents the effect of the entanglements. The elastic free energy of a network in the constrained junction model is the sum of the free energy of the phantom network and the free energy of the constraints. ${ }^{5,6,69}$

$$
\begin{aligned}
A_{\text {elastic }}= & A_{\text {phantom }}+A_{\text {constraint }} \\
= & \xi k_{B} T \sum_{i} \lambda_{i}^{2}+\frac{1}{2} N_{f} k_{B} T \\
& \times \sum_{i}\left[B_{i}+D_{i}-\ln \left(B_{i}+1\right)-\ln \left(D_{i}+1\right)\right],
\end{aligned}
$$

where the summation is over each coordinate axis, $i$, and $\xi$ $=(1-2 / f) N_{c}$, is the cycle rank of the network, i.e., the number of chains that must be cut in order to reduce the network to a tree with no closed cycles. The terms $B_{i}$ and $D_{i}$ are given by

$$
B_{i}=\kappa^{2} \frac{\left(\lambda_{i}^{2}-1\right)}{\left(\lambda_{i}^{2}+\kappa\right)^{2}}, \quad D_{i}=\frac{\lambda_{i}^{2} B_{i}}{\kappa},
$$

where $\lambda_{i}$ is the macroscopic deformation ratio along the coordinate axis, $i$. The parameter $\kappa$ is a measure of the strength of the constraints, and is directly related to the number of crosslinks in the volume of a single network chain, i.e., $\kappa$ $=I\left\langle R_{0}^{2}\right\rangle^{3 / 2} N_{f} / V_{0}$, where $I$ is a constant, and $N_{f}$ is the number of crosslinks in the $f$-functional network. In order to compare the predictions of this theory with our simulation results, we calculated the network pressure as the sum of the elastic component from the above relation (obtained using the relation $P=-\partial A_{\text {elastic }} / \partial V$ ), and the GFD liquid-like contribution to the pressure [Eq. (34)]. The chain meansquared end-to-end distance $\left\langle R_{0}^{2}\right\rangle$ is taken to be equal to the mean-squared end-to-end distance of the network chains, $\left\langle R^{2}\right\rangle$, at $\eta_{0}$, which again takes the value $\eta_{0}=0.45$. The values of $\left\langle R^{2}\right\rangle / \sigma^{2}$ are obtained from Table IV. The value of the constant $I$ is taken to be 1 . [The value of the constant does not materially affect the model performance; this was checked by trying a range of $I$ values from 0.01 to 1000 in Eq. (45).]

Figure 7 shows the constrained junction model prediction for the scaled network pressure, $n P^{*}$, (dotted line) versus the packing fraction, along with the predictions of the non-Gaussian dendrimer model (solid line) and the Obhukov model (dashed line), for the 50-mer and 100-mer networks in the low $\eta$ region. The symbols represent our simulation results. It can be seen that the constrained junction model does not predict the downturn in the pressure at very low packing fractions. At intermediate to high packing fractions, the performance of the constrained junction model prediction for the network pressure is similar to that of the affine model.

The elastic free energy of networks that are solution crosslinked and then deswollen is obtained by Obhukov, $\mathrm{Ru}-$ binstein, and Colby. ${ }^{53}$ As mentioned earlier, the chain configurations in such networks are somewhat collapsed relative to melt-crosslinked networks and contain fewer trapped entanglements. This means that they can undergo very large deformations before rupturing. Since our crosslinking procedure is akin to solution crosslinking followed by deswelling,

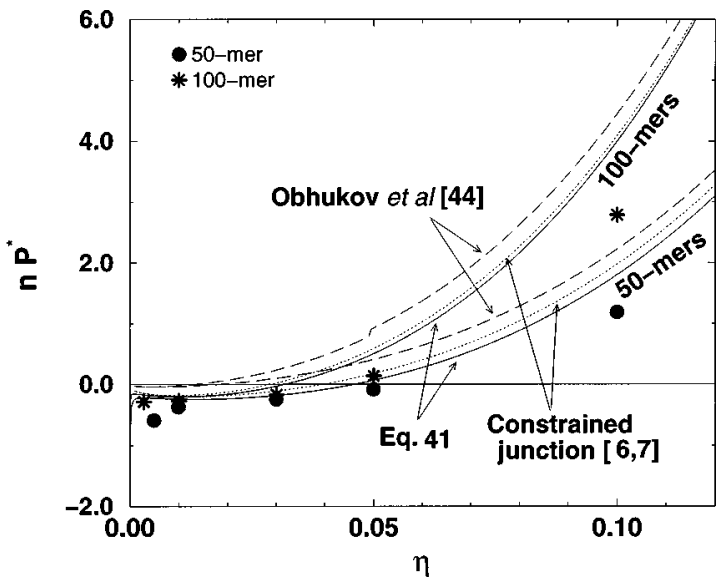

FIG. 7. Comparison of simulation results for the reduced pressure, $P^{*}$, multiplied by the chain length, $n$, vs packing fraction, $\eta$, for the 50-mer and 100-mer trifunctional networks, with theoretical predictions for the network pressure in the region $\eta<0.12$. The elastic components of the pressure are taken from the constrained junction model (Refs. 5,6) (dotted line), the model of Obhukov, Rubinstein, and Colby (Ref. 53) (dashed line), and the non-Gaussian dendrimer-network model (solid line). The liquid-like component of the network pressure is taken from the GFD theory [Eq. (34)] for the constrained junction and Obhukov models and Eq. (41) for the nonGaussian dendrimer-network model).

and our networks therefore contain slightly collapsed chains, the work of Obhukov, Rubinstein, and Colby is of special relevance to us. The underlying idea in their work is as follows: The chains in the deswelled networks form numerous temporary entanglements because of the solution crosslinking and deswelling procedure. When a chain in a deswelled network is stretched, it forms a string of blobs, each blob containing a portion of the chain in a special double-folded configuration. When the chains in a melt-crosslinked network are stretched, they also form a string of blobs, ${ }^{67,60}$ but each blob contains a portion of the chain in a Gaussian configuration.

For deswollen networks, Obhukov, Rubinstein, and Colby predict a linear stress-strain relation in the low deformation regime, a nonlinear relation at intermediate deformations, and a linear relation again at very large deformations. The elastic free energy in the low, intermediate, and large deformation regimes obeys the following relations

$$
\begin{aligned}
& A_{\text {elastic }} \sim N_{c} k_{B} T \lambda^{2}, \quad \lambda\left[R_{0}^{s}\left(\eta_{0}^{s}\right)^{1 / 3}\right]<\left(n_{e} n\right)^{1 / 4} \\
& \sim N_{c} k_{B} T\left[R_{0}^{s}\left(\eta_{0}^{s}\right)^{1 / 3}\right]^{4 / 3}\left(n_{e} n\right)^{-1 / 3} \lambda^{4 / 3}, \\
&\left(n_{e} n\right)^{1 / 4}<\lambda\left[R_{0}^{s}\left(\eta_{0}^{s}\right)^{1 / 3}\right]<n / n_{e}^{1 / 2} \\
& \sim N_{c}\left(\eta_{0}^{s}\right)^{5 / 12} \lambda, \quad \lambda\left[R_{0}^{s}\left(\eta_{0}^{s}\right)^{1 / 3}\right]>n / n_{e}^{1 / 2},
\end{aligned}
$$

where the quantity $R_{0}^{s}\left(\eta_{0}^{s}\right)^{1 / 3}$ represents the end-to-end distance of the chain in the deswollen undeformed network, with $R_{0}^{s}$ and $\eta_{0}^{s}$ being the chain end-to-end distance and polymer volume fraction in the network preparation (solution) state, and $n_{e}$ being the entanglement length. We have attempted to make a qualitative comparison of our simula- 


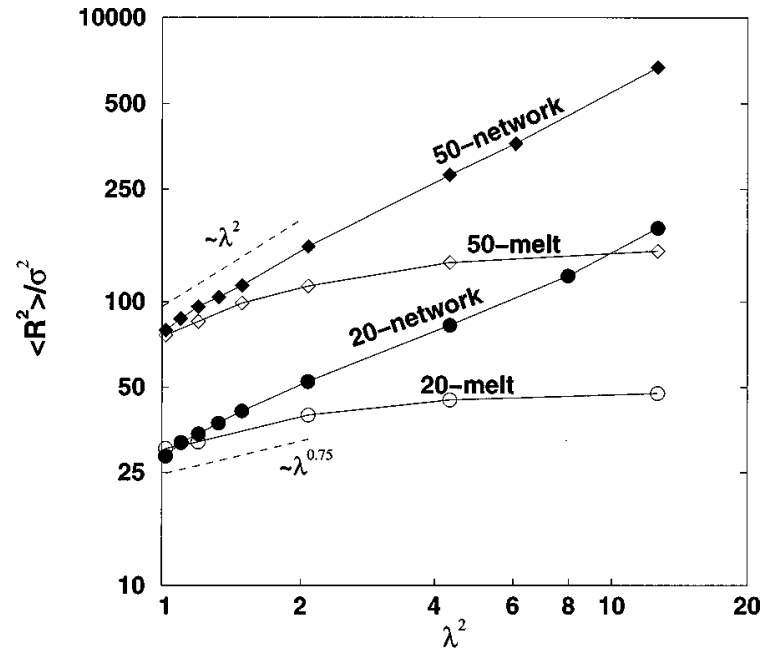

FIG. 8. Simulation results for the mean squared end-to-end distance, $\left\langle R^{2}\right\rangle / \sigma^{2}$, of trifunctional networks (solid symbols) and uncrosslinked chain systems (open symbols) for chain lengths 20 and 50 vs squared deformation ratio, $\lambda^{2}$, on a $\log -\log$ scale. Lines are added solely as guides to the reader's eye.

tion results with the network pressure obtained from the Obhukov model, by calculating the latter as the sum of the elastic component obtained from the above relation, and the GFD liquid-like component [Eq. (34)]. The parameter $n_{e}$ in our calculations is taken to be equal to the chain entanglement length in an equivalent melt at a packing fraction of 0.45 ; based on the article of Smith, Hall, and Freeman, ${ }^{45}$ on the dynamics of melts, $n_{e}=29$. Figure 7 shows the Obhukov model prediction for the scaled pressure, $n P^{*}$, versus the packing fraction, $\eta$, in the low $\eta$ region. It can be seen that the Obhukov model does not predict the pressure downturn at very low packing fractions. The Obhukov model is as successful as the affine model in predicting the network pressure at high to intermediate densities.

It is also of interest to compare the chain dimensions of network chains with those of uncrosslinked linear chains at the same packing fraction. Figure 8 shows the mean-squared end-to-end distance of 20-mer and 50-mer network chains (solid symbols) and uncrosslinked chains (open symbols) versus the squared deformation ratio, $\lambda^{2}$ on a log-log scale. The uncrosslinked system chain dimensions are given in Table V. At $\lambda^{2}=1.0$, i.e., at $\eta=0.45$, the chain dimensions in the crosslinked and uncrosslinked systems are nearly identical; evidently at these high packing fractions, the chain dimensions are virtually unaffected by the system topology. As

TABLE V. Simulation results for the uncrosslinked chain mean-squared end-to-end distance, $\left\langle R^{2}\right\rangle / \sigma^{2}$, at each packing fraction, $\eta$, and corresponding deformation ratio, $\lambda$, at chain lengths $n=20,35,50$, and 100 .

\begin{tabular}{cccccc}
\hline \hline$\eta$ & $\lambda$ & 20 & 35 & 50 & 100 \\
\hline 0.45 & 1.0 & 30.43 & 55.77 & 76.38 & 160.68 \\
0.35 & 1.09 & 32.07 & 60.07 & 85.16 & 168.53 \\
0.25 & 1.22 & $\cdots$ & 66.96 & 99.14 & 217.14 \\
0.15 & 1.44 & 39.84 & 75.25 & 113.80 & 233.15 \\
0.05 & 2.08 & 45.08 & 82.47 & 137.86 & 305.33 \\
0.01 & 3.56 & 47.56 & 97.46 & 150.92 & 349.44 \\
\hline \hline
\end{tabular}

TABLE VI. Simulation results for the reduced pressure, $P^{*}$, at each packing fraction, $\eta$, and corresponding deformation ratio, $\lambda$, for tetrafunctional networks at chain lengths $n=20,35$, and 50 .

\begin{tabular}{ccccc}
\hline \hline$\eta$ & $\lambda$ & 20 & 35 & 50 \\
\hline 0.45 & 1.0 & 5.094 & 5.127 & 5.143 \\
0.35 & 1.09 & 1.717 & 1.751 & 1.753 \\
0.25 & 1.22 & 0.488 & 0.502 & 0.507 \\
0.15 & 1.44 & 0.070 & 0.091 & 0.094 \\
0.05 & 2.08 & -0.0055 & -0.0032 & -0.00055 \\
0.01 & 3.56 & -0.024 & -0.013 & -0.0063 \\
\hline \hline
\end{tabular}

$\lambda^{2}$ increases, the uncrosslinked chain dimensions increase, but much more slowly than the crosslinked chain dimensions; in fact the uncrosslinked chains approximately obey the semidilute solution relation, ${ }^{60}\left\langle R^{2}\right\rangle \propto \eta^{-1 / 4} \propto \lambda^{3 / 4}$ in the region $\lambda^{2}<2$ (approximately), while the crosslinked chains obey the affine relation, $\left\langle R^{2}\right\rangle \propto \lambda^{2}$ in the region $\lambda^{2}<2$ (approximately). For $\lambda^{2}>2$ ( $\eta$ less than about 0.15$)$, the uncrosslinked chain dimensions are nearly independent of the deformation, the reason being that in this very dilute regime the chains form swollen coils that do not interact with each other, and are therefore unaffected by the overall change in system dimensions. The crosslinked chain dimensions continue to increase with $\lambda$ in the $\lambda^{2}>2$ regime, with a nonaffine scaling of $\left\langle R^{2}\right\rangle \propto \lambda^{2 g}$ where $0<g<1$. The exact value of $g$ is dependent on the chain length, for instance, $g$ $=0.682$ for the 20 -mer network, $g=0.775$ for the 35 -mer network, $g=0.826$ for the 50-mer network, and $g=0.841$ for the 100-mer network. Thus the chain dimensions of uncrosslinked chain systems show a much weaker scaling with the deformation ratio than the network chains.

We have also investigated the effect of network functionality on the network pressure and chain end-to-end distances by comparing the behavior of trifunctional and tetrafunctional networks. Tables VI and VII show the reduced pressure and chain dimensions of three tetrafunctional networks of chain length 20,35, and 50. The pressure variation with packing fraction of the tetrafunctional and trifunctional networks is shown in Fig. 9. The inset is a magnification of the low $\eta$ region. The open symbols represent the trifunctional network results and the filled symbols represent the tetrafunctional network results. We see that the pressurevolume behavior of tetrafunctional networks is identical to that of the trifunctional networks. Hence it appears that the pressure-volume behavior of these athermal networks is affected very little by the network functionality. Our theory too predicts a weak dependence on the functionality, both in the liquid-like term and in the elastic term [Eqs. (42) and (43)]. Figure 10 shows the simulation results (symbols) and nonGaussian dendrimer-network theory predictions for the scaled pressure, $n P^{*}$, versus the packing fraction, $\eta$, for a trifunctional and tetrafunctional network, both of chain length 20 . Our theory predicts that the tetrafunctional network pressure follows the same trend as the trifunctional network pressure, which is in agreement with our simulation results.

Figure 11 shows the variation in the mean-squared endto-end distances of the tetrafunctional and trifunctional net- 


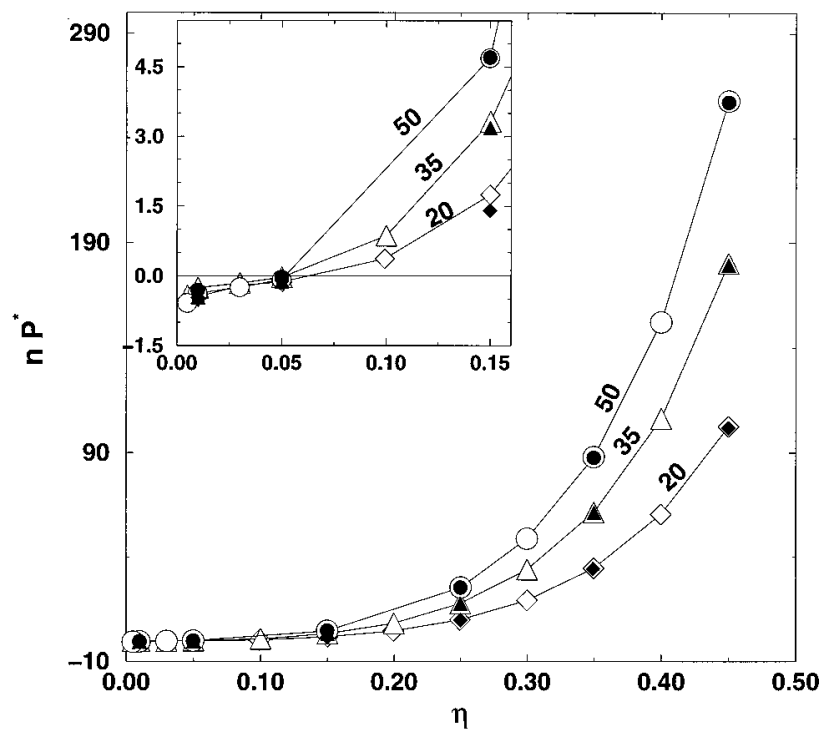

FIG. 9. Simulation results for the reduced pressure, $P^{*}$, multiplied by the chain length, $n$, vs the packing fraction, $\eta$, for trifunctional (larger open circles) and tetrafunctional networks (smaller filled circles), for chain lengths 20,35, and 50. The inset shows the pressure in the low density region. Lines are added solely as guides to the reader's eye.

work chains with deformation ratio on a log-log scale. As before, the closed symbols represent the tetrafunctional network results and the open symbols represent the trifunctional network results. The dimensions of the tetrafunctional network chains appear to be approximately the same as the dimensions of the trifunctional network chains at all deformation ratios, with the exception that the 20-mer tetrafunctional network chains are slightly collapsed relative to the corresponding trifunctional network chains at $\lambda^{2}$ close to 1.0 (high packing fractions). Thus it appears that the network chain dimensions are almost unaffected by the functionality at all packing fractions.

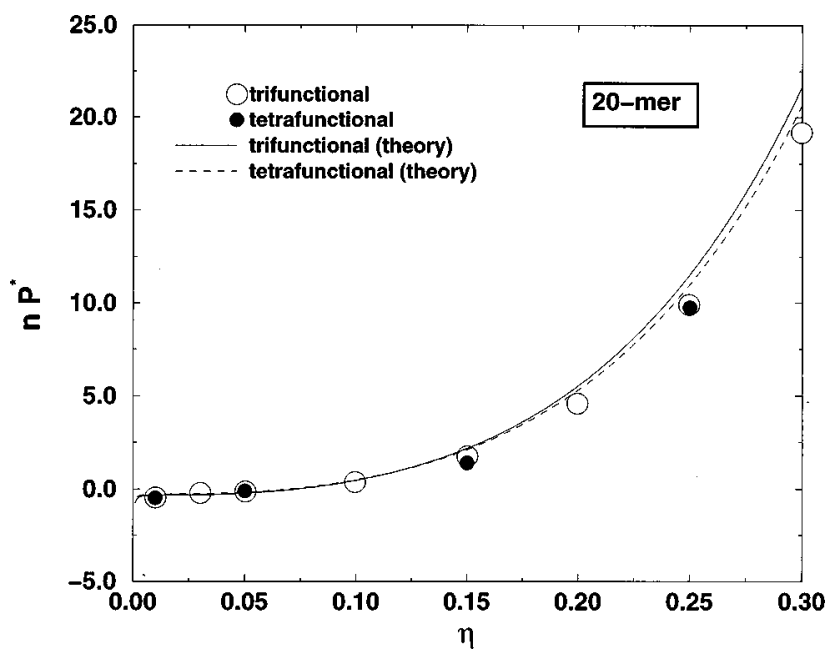

FIG. 10. Simulation results (symbols) and non-Gaussian dendrimer-network model theoretical predictions (lines) for the reduced pressure, $P^{*}$, multiplied by the chain length, $n$, vs the packing fraction, $\eta$, for a trifunctional network and a tetrafunctional network, both of chain length 20 .
TABLE VII. Simulation results for the chain mean squared end-to-end distance, $\left\langle R^{2}\right\rangle / \sigma^{2}$, at each packing fraction, $\eta$, and corresponding deformation ratio, $\lambda$, for tetrafunctional networks at chain lengths $n=20,35$, and 50 .

\begin{tabular}{ccccc}
\hline \hline$\eta$ & $\lambda$ & 20 & 35 & \multicolumn{1}{c}{50} \\
\hline 0.45 & 1.00 & 26.11 & 56.31 & 81.24 \\
0.35 & 1.09 & 30.69 & 63.02 & 96.31 \\
0.25 & 1.22 & 36.72 & 78.46 & 116.80 \\
0.15 & 1.44 & 47.21 & 104.47 & 162.03 \\
0.05 & 2.08 & 74.46 & 183.61 & 285.19 \\
0.01 & 3.56 & 177.88 & $\cdots$ & 686.06 \\
\hline \hline
\end{tabular}

\section{CONCLUSION}

Discontinuous molecular dynamics simulations were conducted in order to investigate the pressure-volume properties of near-perfect trifunctional and tetrafunctional networks. A simple analytical equation of state is presented in which the network chains are treated in two ways; (1) as having a Gaussian end-to-end vector distribution (the Gaussian dendrimer-network model), and (2) as having a nonGaussian end-to-end vector distribution (the non-Gaussian dendrimer-network model). The predictions of the Gaussian dendrimer-network model for the network pressure are similar to those of the Flory affine model; both models accurately predict the network pressure over the range of packing fractions from $\eta=0.45$ to $\eta \sim 0.1$, and fail at low packing fractions $(\eta<0.1)$. The non-Gaussian dendrimer-network model is able to predict the network pressure over the entire range of packing fractions studied, and successfully predicts the observed downturn in network pressure at very low packing fractions, $\eta \sim 0.003$.

A novel algorithm that utilizes the variable bond-length feature of the Bellemans chain was used to perform network volume changes. The efficiency of this algorithm coupled with its applicability to different system topologies allowed us to investigate the pressure-volume behavior of trifunctional and tetrafunctional networks over a wide range of network packing fractions. The simplicity of the algorithm also makes it an attractive tool for NPT or Gibbs ensemble simu-

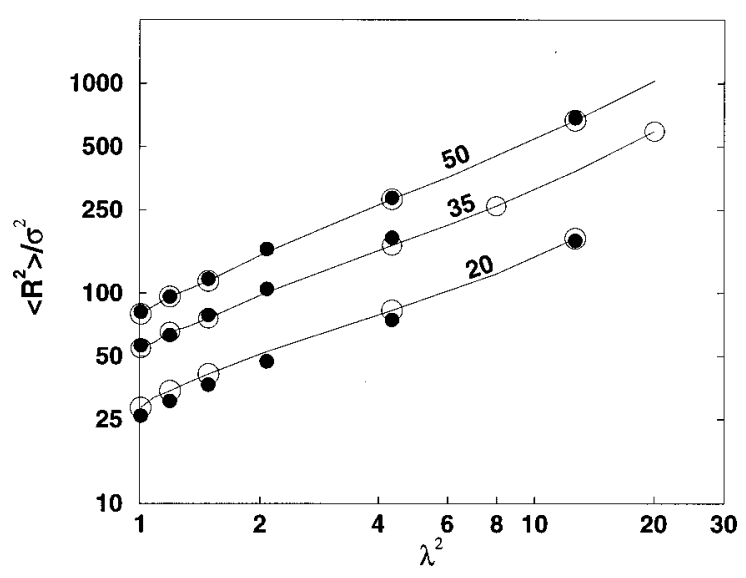

FIG. 11. Simulation results for the mean-squared end-to-end distance, $\left\langle R^{2}\right\rangle / \sigma^{2}$, of trifunctional (larger open circles) and tetrafunctional (smaller closed circles) networks for chain lengths 20, 35, and 50, vs squared deformation ratio, $\lambda^{2}$, on a $\log -\log$ scale. Lines are added solely to guide the reader's eye. 
lations of systems with complex molecular structure, for which conventional volume change moves cannot be used.

As mentioned above, we observed a downturn in the network pressure as the network is expanded to very low packing fractions. This occurs when the network chains are extended to between $60 \%$ and $70 \%$ of their maximum extended length. At these large extensions, the probability distribution of the chain end-to-end vectors is not expected to follow the Gaussian form. In fact, we found that the nonGaussian dendrimer-network equation of state based on the non-Gaussian distribution suggested by Kuhn and Grun ${ }^{43}$ [Eq. (33)] was remarkably successful in predicting this drop in pressure. The success of Eq. (33) does not necessarily imply that the non-Gaussian probability distribution of Kuhn and Grun is accurate, but it does suggest that network equations of state that account for the finite extensibility of polymer chains can successfully predict network pressurevolume behavior at low packing fractions.

Comparison of the chain end-to-end vectors in trifunctional networks and uncrosslinked chain systems shows that at very high packing fractions, the network chain dimensions are approximately equal to the uncrosslinked chain dimensions. This is due to the fact that at very high packing fractions, the chains are so densely packed together that their dimensions are unaffected by the specific topology of the system. As the packing fraction decreases, the chain dimensions begin to be influenced by the system topology and the network chain dimensions are observed to increase rapidly relative to the uncrosslinked system chain dimensions. We also investigated the pressure-volume behavior and the chain dimensions of tetrafunctional networks, and found that network pressure and chain dimensions are minimally affected by the functionality.

\section{ACKNOWLEDGMENTS}

This work was supported by the GAANN Computational Sciences Fellowship of the U.S. Department of Education, and the Office of Energy Research, Basic Sciences, Chemical Science Division of the U.S. Department of Energy under Contract No. DE-FG05-91ER14181. Acknowledgement is made to the Donors of the Petroleum Research Fund administered by the American Chemical Society for partial support of this work. The authors thank the North Carolina Supercomputing Center for grants of CPU time on the Cray Y-MP and T90, and on the workstation cluster.

\section{APPENDIX}

Here we show that the effective spring constant of the dendritic structure displayed in Fig. 1 can be obtained in terms of the spring constant of a single chain (branch) of the structure, in the following form:

$$
k_{\text {dendrimer }}=k_{\text {chain }} f(f-2)\left[\frac{(f-1)^{(m-1)}}{(f-1)^{m}-1}\right],
$$

where $m$ is the rank of the dendrimer. If the dendrimer chains are ideal and Gaussian, $k_{\text {chain }}=3 k_{B} T /\left\langle R_{0}^{2}\right\rangle$.

To show how Eq. (A1) is obtained, we invoke the analogy between the elasticity of a perfect polymer network and the conductance of an electrical resistor network. ${ }^{10,60}$ For an ideal chain, the magnitude of the elastic restoring force exerted by the chain is given by $F_{\text {chain }}=-k_{\text {chain }} R$. The sum of the forces in any direction $i$ exerted on a given junction in the network by the chains attached to that junction must be zero, i.e.,

$$
\sum_{i} F_{\text {chain }}^{i}=0
$$

This is analogous ${ }^{10,60}$ to the Kirchoff law for electrical circuits, which states that the net current entering a given node in the circuit must be zero. Comparing the ideal chain relation, $F_{\text {chain }}=-k_{\text {chain }} R$, and the electrical resistance relation (Ohm's law), $i=\Delta V / R$, where $i$ is the current, $R$ is the resistance and $\Delta V$ is the voltage drop across the resistor, we see that analogies can be drawn between: (1) the spring constant, $k_{\text {chain }}$ and the conductance, $1 / R,(2)$ the magnitude of the force, $F_{\text {chain }}$ and the current, $i$, and (3) the chain end-toend distance, $R$, and the voltage drop, $\Delta V$.

We now want to use simple electrical circuit laws to obtain the effective resistance, $R_{\text {dendrimer }}$, of the dendritic network shown in Fig. 1, when each branch (chain) of the dendrimer is replaced by a resistance, $R=1 / k_{\text {chain }}$. As described in Sec. III, the $f$ functional dendrimer network in Fig. 1 consists of concentric layers of branches or resistors, with the first layer containing $f$ branches or resistors, the second layer containing $f(f-1)$ branches or resistors, the third layer containing $f(f-1)^{2}$ branches or resistors, and so on. Now consider that the outermost nodes of the dendrimer are grounded, and that a current $i$ enters the network at $O$. (This is analogous to applying a force $F$ across the network). Then the sum of the voltage drops over any path taken by the current between the point $O$ and the grounded nodes is equal to the overall voltage drop, $\Delta V$, between $O$ and ground. The effective resistance of the network, $R_{\text {dendrimer }}$ is then simply given by $R_{\text {dendrimer }}=\Delta V / i$. Due to the symmetry of the network, all branches in the same layer will have the same voltage drop across them and the same current flowing through them. Hence by simple symmetry arguments, the resistors in the first layer of the network will have a current i/f flowing through them, the resistors in the second layer will have a current $i / f(f-1)$ flowing through them, and so on. The voltage drop across any one of the resistors in the first layer of the network is then $i R / f$, the voltage drop across any one of the resistors in the second layer of the network is $i R / f(f-1)$, and so on. By summing the voltage drops over all the concentric layers of the network, we can calculate the total voltage drop across the network

$$
\begin{aligned}
\Delta V=i R_{\text {dendrimer }}= & \frac{i}{f} R+\frac{i}{f(f-1)} R+\frac{i}{f(f-1)^{2}} R+\cdots \\
& +\frac{i}{f(f-1)^{m-1}} R
\end{aligned}
$$

where $m$ represents the rank or the number of layers in the network. The right hand side of the above equation is a geometric series in $1 /(f-1)$, and can be summed in order to obtain the effective resistance of the dendrimer network 


$$
R_{\text {dendrimer }}=\frac{R}{f}\left[\frac{1-\frac{1}{(f-1)^{m}}}{1-\frac{1}{(f-1)}}\right]=\frac{R}{f(f-2)}\left[\frac{(f-1)^{m}-1}{(f-1)^{(m-1)}}\right] \text {. }
$$

Since the resistance is inversely related to the spring constant, the effective spring constant of the dendrimer can be written as

$$
k_{\text {dendrimer }}=k_{\text {chain }} f(f-2)\left[\frac{(f-1)^{(m-1)}}{(f-1)^{m}-1}\right] .
$$

${ }^{1}$ P. J Flory and J. Rehner, J. Chem. Phys. 11, 512 (1943).

${ }^{2}$ F. T. Wall, J. Chem. Phys. 11, 527 (1943).

${ }^{3}$ H. M. James and E. Guth, J. Chem. Phys. 11, 455 (1943).

${ }^{4}$ H. M. James and E. Guth, J. Chem. Phys. 15, 669 (1947).

${ }^{5}$ P. J. Flory and B. Erman, Macromolecules 15, 800 (1982).

${ }^{6}$ B. Erman and P. J. Flory, Macromolecules 15, 806 (1982).

${ }^{7}$ G. Marrucci, Rheol. Acta 18, 193 (1979).

${ }^{8}$ R. C. Ball, M. Doi, S. F. Edwards, and M. Warner, Polymer 22, 1010 (1981).

${ }^{9}$ S. F. Edwards and T. A. Vilgis, Rep. Prog. Phys. 51, 243 (1988).

${ }^{10}$ P. G. Higgs and R. C. Ball, Europhys. Lett. 8, 357 (1989).

${ }^{11}$ T. A. Vilgis and F. Boue, Polymer 27, 71 (1986).

${ }^{12}$ T. A. Vilgis and B. Erman, Macromolecules 26, 6657 (1993).

${ }^{13}$ T. A. Vilgis and G. Heinrich, Phys. Rev. E 49, 2167 (1994).

${ }^{14}$ J. F. Douglas and G. B. McKenna, Macromolecules 26, 3282 (1993).

${ }^{15}$ R. Everaers, Eur. Phys. J. B 4, 341 (1998).

${ }^{16}$ D. Adolf, Macromolecules 21, 228 (1988).

${ }^{17}$ M. Kosc, Colloid Polym. Sci. 266, 266 (1988).

${ }^{18} \mathrm{G}$. Marrucci, Macromolecules 14, 434 (1981).

${ }^{19}$ W. W. Graessley, Adv. Polym. Sci. 47, 68 (1982).

${ }^{20}$ S. F. Edwards, Br. Polym J. 17, 123 (1985).

${ }^{21}$ R. J. Gaylord, Polym. Bull. 8, 325 (1982).

${ }^{22}$ R. J. Gaylord and J. F. Douglas, Polym. Bull. 18, 347 (1987).

${ }^{23}$ J. Gao and J. H. Weiner, Macromolecules 20, 2520 (1987).

${ }^{24}$ J. Gao and J. H. Weiner, Macromolecules 20, 2525 (1987).

${ }^{25} \mathrm{~J}$. H. Weiner and J. Gao, in Molecular Basis of Polymer Networks, edited by A. Baumgartner and C. E. Picot (Springer, New York, 1989).

${ }^{26}$ E. R. Duering, K. Kremer, and G. Grest, Phys. Rev. Lett. 67, 3531 (1991).

${ }^{27}$ E. R. Duering, K. Kremer, and G. S. Grest, Prog. Colloid Polym. Sci. 90, 13 (1992).

${ }^{28}$ G. S. Grest, K. Kremer, and E. R. Duering, Physica A 194, 330 (1993).

${ }^{29}$ E. R. Duering, K. Kremer, and G. Grest, J. Chem. Phys. 101, 8169 (1994).

${ }^{30}$ N. R. Kenkare, S. W. Smith, C. K. Hall, and S. A. Khan, Macromolecules 31, 5861 (1998).

${ }^{31}$ H. L. Trautenberg, J. U. Sommer, and D. Goritz, J. Chem. Soc., Faraday Trans. 91, 2649 (1995).
${ }^{32}$ F. A. Escobedo and J. J. de Pablo, J. Chem. Phys. 104, 4788 (1996).

${ }^{33}$ F. A. Escobedo and J. J. de Pablo, J. Chem. Phys. 106, 793 (1997).

${ }^{34}$ F. A. Escobedo and J. J. de Pablo, Mol. Phys. 90, 437 (1997).

${ }^{35}$ J. U. Sommer, Macromol. Symp. 81, 139 (1994).

${ }^{36}$ N. Rennar and W. Opperman, Colloid Polym. Sci. 270, 527 (1992).

${ }^{37}$ M. A. Sharaf, J. E. Mark, and A. S. Alshamsi, Polym. J. (Tokyo) 28, 375 (1996).

${ }^{38}$ M. A. Sharaf and J. E. Mark, Polymer 35, 740 (1994).

${ }^{39}$ P. H. Sung and J. E. Mark, Polym. J. (Tokyo) 12, 835 (1980).

${ }^{40}$ R. Dickman and C. K. Hall, J. Chem. Phys. 85, 4108 (1986).

${ }^{41}$ C. K. Hall, M. A. Denlinger, and K. G. Honnell, Fluid Phase Equilibria 53, 151 (1989).

${ }^{42}$ K. G. Honnell and C. K. Hall, J. Chem. Phys. 90, 1841 (1989).

${ }^{43}$ W. Kuhn and F. Grun, Kolloid-Z. 101, 248 (1942).

${ }^{44}$ L. R. G. Treloar, The Physics of Rubber Elasticity (Clarendon, Oxford, 1958).

${ }^{45}$ S. W. Smith, C. K. Hall, and B. D. Freeman, J. Chem. Phys. 104, 5616 (1996).

${ }^{46}$ S. W. Smith, C. K. Hall, and B. D. Freeman, J. Comput. Phys. 134, 16 (1997).

${ }^{47}$ D. C. Rapaport, J. Phys. A 11, L213 (1978).

${ }^{48}$ D. C. Rapaport, J. Chem. Phys. 71, 3299 (1979).

${ }^{49}$ A. Bellemans, J. Orban, and D. V. Belle, Mol. Phys. 39, 781 (1980).

${ }^{50}$ B. J. Alder and T. E. Wainwright, in Symposium on Trans. Processes In Stat. Mech., edited by I. Prigogine (Interscience, New York, 1956).

${ }^{51}$ B. J. Alder and T. E. Wainwright, J. Chem. Phys. 31, 459 (1959).

${ }^{52}$ J. M. Haile, Molecular Dynamics Simulation: Elementary Methods (Wiley, New York, 1992).

${ }^{53}$ S. P. Obukhov, M. Rubinstein, and R. H. Colby, Macromolecules 27, 3191 (1994).

${ }^{54}$ J. Scanlan, J. Polym. Sci. 43, 501 (1960).

${ }^{55}$ D. S. Pearson and W. W. Graessley, Macromolecules 11, 528 (1977).

${ }^{56}$ G. Gee, J. B. M. Herbert, and R. C. Roberts, Polymer 6, 541 (1965).

${ }^{57}$ B. E. Eichinger and N. A. Neuburger, in Biological and Synthetic Polymer Networks, edited by O. Kramer (Elsevier Applied Science, London, 1986).

${ }^{58}$ J. A. Duiser and A. J. Staverman, in Physics of Non-Crystalline Solids, edited by J. A. Prins (North-Holland, Amsterdam, 1965).

${ }^{59}$ W. W. Graessley, Macromolecules 8, 186 (1975).

${ }^{60}$ P. G. de Gennes, Scaling Concepts in Polymer Physics (Cornell U.P., Ithaca, NY, 1979).

${ }^{61}$ P. J. Flory, Principles of Polymer Chemistry (Cornell U.P., Ithaca, 1953).

${ }^{62}$ N. F. Carnahan and K. E. Starling, J. Chem. Phys. 51, 635 (1969).

${ }^{63}$ D. J. Tildesley and W. B. Streett, Mol. Phys. 41, 85 (1980).

${ }^{64}$ A. Yethiraj and C. K. Hall, J. Chem. Phys. 94, 3943 (1991).

${ }^{65}$ J. Alejandre and G. A. Chapela, Mol. Phys. 61, 1119 (1987).

${ }^{66}$ A. L. Andrady, M. A. Llorente, and J. E. Mark, J. Chem. Phys. 72, 2282 (1980).

${ }^{67}$ P. Pincus, Macromolecules 9, 386 (1976).

${ }^{68}$ G. Ronca and G. Allegra, J. Chem. Phys. 63, 4990 (1975).

${ }^{69}$ J. E. Mark and B. Erman, Rubberlike Elasticity: A Molecular Primer (Wiley-Interscience, New York, 1988). 